\title{
Evaluating Müller-Lyer effects using single fin-set configurations
}

\author{
ERNEST GREENE and BRIAN NELSON \\ University of Southern California, Los Angeles, California \\ and Neuropsychology Foundation, Los Angeles, California
}

\begin{abstract}
Four experiments were conducted to study the bias of perceived length for Müler-Lyer configurations that contained a single set of fins (i.e., two segments that join to form a vertex). The experiments manipulated several factors that have been shown to be critical to the effect: (1) version (which way the apex pointed), (2) length of the stimulus span, (3) presence or absence of a line segment in the span being judged, (4) fin length, (5) fin angle, and (6) the zone in which the response was rendered. Using percent error as the index of perceptual distortion, the major finding was that the two versions show an opposite slope for strength of effect as a function of span. When stimulus spans were plotted against response means (not converting to percent error), an almost perfect linear relation was found. These results indicate that the perceptual effects can be modeled as a linear system having two parameters through which the treatments exert their influence. The results are discussed in relation to major theories of mechanism for the Müller-Lyer illusion.
\end{abstract}

Müller-Lyer (1889) has provided us with one of the bestknown and most heavily investigated geometric illusions. His classic configurations are shown in Figure 1, along with a number of other versions. These variants clearly demonstrate that arrowheads, tailfeathers, and related elements can distort the perceived length of line segments and distances in visual space itself. The diversity of alternative designs shows that the metric distortion does not pivot on a narrow stimulus characteristic. Substantial distortion is present when the arrowheads and tailfeathers are replaced by circles or when a single fin is used at each end of a shaft. Many investigators have shown that the effect survives deletion of elements, with Greist-Bousquet and Schiffman (1981b) providing what may be the ultimate demonstration of that fact. They eliminated the shaft, placed dots at the end of the span to be judged, and attached only a single fin to one of the dots. They were able to quantify a bias of perceived span, contraction when the fin was tilted to lie over the span to be judged and expansion when it was tilted away.

Finding that one can get metric distortion with a reduced set of elements is of great importance for unraveling the nature of the Müller-Lyer effect, both in identifying the most critical factors and in testing theories. Study of a version that has fewer elements greatly simplifies the problem of experimental control, especially if the goal is to quantify the influence of length, angle, or other characteristics of the configuration. One may wish to evaluate just

This research was supported in part by the Neuropsychology Foundation, Los Angeles. The authors thank Jozsef Fiser for his help in modeling the data. Correspondence should be addressed to E. Greene, Department of Psychology, University of Southern California, Los Angeles, CA 90089 (e-mail: egreene@bcf.usc.edu). a few different lengths of each component and, perhaps, fin angle. Even with a figure as simple as the classic version by Müller-Lyer (1889), the number of permutations that must be created and tested becomes formidable.

Additionally, the study of versions with a reduced set of elements is essential in the formulation of theory. For example, one might favor a neuroreductionist view, wherein the length of line segments is judged on the basis of output from orientation-selective neurons in primary visual cortex. It is known that some neurons have "end-stopping" inhibition and thus respond preferentially to segments that do not extend into these zones. Building on this approach, the action of the arrowheads or tailfeathers might be modeled in terms of differential encroachment into the end-stopping zones. Perhaps the signal that indicates the segment's length is modified, or there is a change of dominance among the cells that preferentially respond to various lengths. Being made aware that there is substantial distortion in a version that has no shaft greatly dampens one's enthusiasm for such a model. At the very least, an advocate of this approach would need to find evidence that said neurons are stimulated by the empty space that lies between a set of fins.

We profit, therefore, from many prior studies that have used Müller-Lyer variants to identify factors that are critical for metric distortion. These are cited subsequently, but, in the interest of efficiency, let us say what has not been answered by the previous work. We are struck by how litthe is known about how a given distortion-inducing element (e.g., a fin set) influences the adjacent space. Is there a systematic change in the strength of distortion at various distances from the fin set? If so, is the metric of space changed by the addition or subtraction of a constant amount? Alternatively, is the distance multiplied by a constant, or is there a geometric gradient of effect as the dis- 


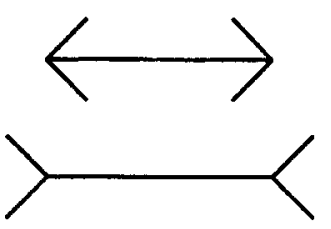

$\mathbf{B}$

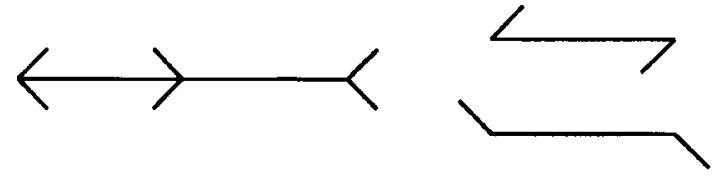
C
D

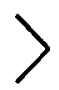
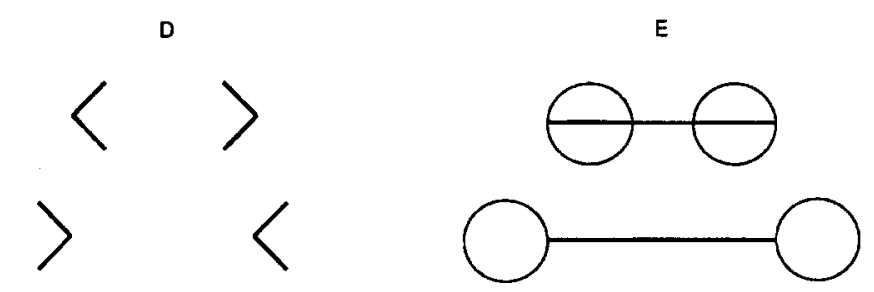

Figure 1. Various versions of the Müller-Lyer: (A) the classic configurations first presented by Müller-Lyer (1889); (B) the composite version (Brentano, 1892; Müller-Lyer, 1889); (C) version tested by Heymans (1896); (D) a version with no shafts (Brentano, 1892); (E) dumbbell version (Delboeuf, 1892).

tance is increased? We can find little evidence that speaks to these issues.

One might think that prior studies of "shaft length" would have answered these questions. In fact, the data are meager, in that researchers have concentrated on other variables or have failed to separate shaft length as a distinct treatment condition. Moreover, most studies have asked for judgment of a shaft (or of an open span) that lies between a pair of fin sets. This kind of configuration cannot provide a definitive answer to the questions posed above. It is conceivable that a single fin set might produce a weak gradient of effect upon the metric of space; however, with a fin set at each end of the span to be judged, the effect might be multiplied and rendered uniform. Thus, the study of strength of effect as a function of distance is best answered using only a single fin set.

In the section that follows, we review the literature to identify the factors that have proven to be critical for the distortion effect. We then present several experiments that examine the bias produced by single fin sets. Each experiment manipulated the span to be judged along with several other variables. Following these experiments, we attempt formal modeling of the metric effects. Finally, in the Discussion section, we evaluate how the results relate to various theories of distortion mechanism.

\section{KEY VARIABLES FOR METRIC DISTORTION}

The most important factor in the Müller-Lyer effect is the orientation of the fin set (i.e., whether the apex points toward or away from the span to be judged). A majority of the quantitative work has specified the angle of each fin in relation to the shaft (or the axis of the span if a shaft is not present). Thus, when each fin is oriented at less than $90^{\circ}$, the configuration is described as an acute version; when it is greater than $90^{\circ}$, it is called an obtuse version. This convention can lead to problems, since these terms can become confused with a treatment condition that manipulates the specific angle of the fins. Thus, in describing a treatment effect for "angle," is one referring to the plot of values across the specific angles, or an overall effect for the acute versus obtuse versions? Furthermore, to compare effects, one must present the corresponding acute and obtuse values on the abscissa of each graph and keep the correspondence in mind when discussing the similarities and differences.

It would be better to have a more general term for the two versions, and we think the geometric concept of concave and convex will serve the purpose. Thus, for a concave version of the Müller-Lyer, the fins tend to surround the zone whose span is judged; for a convex version, they fold away. Thus, concavity and convexity of a given fin set is defined with respect to the adjacent span to be judged, as though an observer were positioned on the span, looking toward the fin set. More formally, a fin set is said to be concave if the angle between the two fins is less than $180^{\circ}$ (measuring on the side that borders the span), and a fin set is designated as convex if the angle is greater than that value. With the new terminology, one can specify a treatment condition having two levels of version (i.e., concave and convex) and thus minimize the potential for confusion with a treatment that manipulates "fin angle."

It is generally thought that a concave version of the illusion produces contraction of distance estimates, and the convex version produces expansion. While one might expect that the metric distortion in each direction would be comparable for the two versions, more often it has been found that the strength of effect is asymmetrical. A number of laboratories have reported that the convex ver- 
sion produces a significantly stronger distortion (Adam \& Bateman, 1983; Bross, Blair, \& Longtin, 1978; Butchard \& Pressey, 1971; Christie, 1975; Cooper \& Runyon, 1970; Day \& Dickinson, 1976; Erlebacher \& Sekuler, 1974; Greist-Bousquet \& Schiffman, 1981a; Sekuler \& Erlebacher, 1971). Though this finding is reasonably robust, there have been instances when the relationship was the reverse (i.e., with the concave version being stronger than the convex; Coren \& Porac, 1983). Also, some investigators report little or no asymmetry between the two versions (Jordan \& Uhlarik, 1986; Worrall \& Firth, 1974). It is possible that asymmetry depends not only on which version is being tested but on how it is tested. Thus, in evaluating the experiments that follow, the data will be examined to determine whether judgment of a single fin set results in asymmetry of effect.

A number of investigators have posited that the magnitude of illusion is dependent on the ratio of fin length to shaft length. For example, Heymans (1896) used a shaft length of $75 \mathrm{~mm}$ (equivalent visual angle not available) and found that distortion was greatest when fin length was $25-30 \mathrm{~mm}$, thus being maximal when the fin/shaft ratio was approximately $30 \%-40 \%$. Nakagawa (1958) studied concave and convex configurations that had a shaft length of $45 \mathrm{~mm}$ (approximately $5 \mathrm{deg}$ of visual angle). Fin lengths in the range of $10-22 \mathrm{~mm}(1.1-2.5 \mathrm{deg})$ were evaluated, and maximal effects were found for the $16 \mathrm{~mm}$ (1.8 deg) fins. This suggests an optimal fin/shaft ratio of $36 \%$. Goryo, Robinson, and Wilson (1984) evaluated shaft lengths that varied from 90 to $110 \mathrm{~mm} \mathrm{(4.5-}$ $5.5 \mathrm{deg}$ ) and with fin $/ \mathrm{shaft}$ ratios of $10 \%, 25 \%$, and $40 \%$. For concave and convex configurations alike, they found the greatest distortion with a fin/shaft ratio of $40 \%$. This is in the same range as that reported by Nakagawa (1958) and Heymans (1896).

Fin angle has been intensively studied. Heymans (1896) varied fin angle in the composite version and found a monotonic decline of distortion effect as the angle between the fin and the shaft became more orthogonal. Similar effects were noted by Lewis (1909), Dewar (1967), and Pressey and Martin (1990). Other laboratories have found a curvilinear relation, wherein the distortion reached a maximum at an intermediate angle (e.g., $45^{\circ}$ or $60^{\circ}$ ) and then decreased as one approached the orthogonal position (Auerbach, 1894; Brentano, 1892; Nakagawa, 1958; Restle \& Decker, 1977). Often, the differential was a function of the version and/or the length of fins (see Coren, 1986; Day \& Dickinson, 1976; Erlebacher \& Sekuler, 1969; Sekuler \& Erlebacher, 1971).

A number of investigators have tested Müller-Lyer configurations in which various line segments were not present but were implied by the positioning of dots (i.e., at locations that corresponded to the ends of the shaft and/or fin segments). For example, Coren (1970) and GreistBousquet and Schiffman (1981a) tested versions that consisted entirely of dots. The overall magnitude of the metric distortion was reduced with these configurations, but they still observed contraction for concave versions and expansion for convex versions. Similar results were re- ported by Beagley (1985) and Greist-Bousquet and Schiffman (1981b). In the work that follows, we have studied whether the distortion differs when the shaft is present or absent and describe this as a manipulation of "shaft state."

In general, then, previous studies suggest that the Müller-Lyer illusion is controlled by five major factors: (1) the version being used (concave/convex), (2) fin length, (3) shaft state (present/absent), (4) stimulus span (the distance to be judged), and (5) fin angle. The first four factors were evaluated in the first experiment, reserving fin angle for the second experiment.

In the experiments described below, metric distortion for a given configuration was produced by a single fin set, consisting of two line segments which joined to form a vertex. The span to be judged was designated as the stimulus span; a dot was provided at each end of this span to delineate it and allow for a uniform specification of task demands whether or not a shaft was present. One of the stimulus dots was always coincident with the vertex of the fin set and, thus, was designated as the vertex dot. The other stimulus dot was described as the reference dot.

Subjects used an operant method to register their perception of the stimulus span. They designated a response span by using one of the stimulus dots as an anchor, and marking a response dot on the page at the distance that was judged to be equal to the stimulus span. (Whether the vertex dot or the reference dot was used as the anchor varied across experiments, as described below.)

In all work reported below, the stimulus dimensions and distances are reported as visual angle in degrees and minutes. These terms are abbreviated as deg and min, respectively, to avoid confusion with rotational angle, which is specified using the conventional symbol (e.g., $45^{\circ}$ ).

\section{EXPERIMENT 1}

The purpose of Experiment 1 was to determine, first of all, how the Müller-Lyer effect would be manifested when only a single fin set was present and the subject was required to judge the span on one side of the fins by selecting a corresponding distance on the other side. This is similar in concept to the demands of the composite configuration, which was studied quantitatively a century ago (Heymans, 1896; Biervliet, 1896, as reviewed by Restle \& Decker, 1977). However, the traditional composite version also has fins at each end of the segments being judged, so we had no assurance that the single fin set would produce classic effects. The primary goal, therefore, was to determine whether the response span would show contraction when the stimulus span was on the concave side of the fin set and expansion when it was on the convex side.

For convenience, we flatly describe the illusory bias as contraction or expansion of the stimulus span. This rather simplistic terminology has the benefit of brevity but should not silence serious questions regarding the locus of action. This includes the question of whether the zone which is used for the response is also subject to a biasing influence. We plan to address this question in the Discussion section. 
Given that the classic metric distortions were not assured with a single fin set, it seemed prudent to examine each of the other factors that are thought to be critical to the effect. Therefore, we varied the span to be judged, the length of the fins, and shaft state (present/absent). To prevent development of a response bias, the position of the span to be judged was varied to the left and right of the page as well. The permutation of five task conditions provided a risk that the results would be difficult to interpret, but it was hoped that some of the variables would prove to be nonsignificant, thus allowing a simpler statistical model to be applied.

\section{Method}

Stimulus materials. The stimulus configurations were printed on $8.5 \times 11$ in. paper with an Apple LaserWriter. A given configuration might contain one of three stimulus elements: (1) a line segment that extended across the horizontal axis, bisecting the page, (2) a pair of dots that were placed on the horizontal axis, with one being positioned at the center of the page, and (3) a fin set consisting of two line segments, each being oriented at $45^{\circ}$ from the horizontal axis and having one end touching the central dot. Line thickness (width) was measured with a dissecting microscope that contained a calibrating reticule; segments had a thickness of 4 min of visual angle, and dots were $8 \mathrm{~min}$ in diameter. Figures $2 \mathrm{~A}-2 \mathrm{D}$ illustrate typical configurations; a given stimulus page would show only a single configuration. Except for the control condition described below, the stimulus always contained a fin set and vertex dot (which were centered on the page), as well as a reference dot (which was positioned to one side). Figure $2 \mathrm{E}$ shows that the subject would register the response span by placing a mark on the side of the page that was opposite the reference dot (shown by an " $x$ " in the illustration).

The stimulus set contained a page for each permutation of the experimental treatments, with two levels of version (concave/convex), two levels of shaft state (present/absent), four levels of stimulus span $(1,2,4$, and $8 \mathrm{deg})$, four levels of fin length $(1,2,4$, and $8 \mathrm{deg})$, and two levels of side. The control configurations consisted of two stimulus dots, one that was centered on the page and one positioned laterally on the horizontal axis of the page at the specified stimulus span. Thus, the no-shaft control condition varied the four levels of stimulus span by the two levels of side. Each stimulus configuration was presented twice, for a total stimulus set of 144 pages. A brief summary of treatment conditions are as follows:

Version. For all configurations of Experiment 1 , each fin was oriented at $45^{\circ}$ from the horizontal axis, as illustrated in Figure 2. For concave versions, each member of the fin set was tipped toward the reference dot (see Figures $2 \mathrm{~A}$ and $2 \mathrm{D}$ ). For convex versions, they were tipped away (Figures $2 \mathrm{~B}$ and $2 \mathrm{C}$ ).
A

B

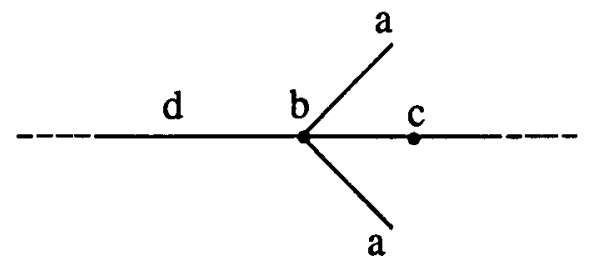

C

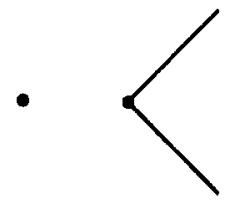

D

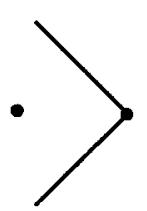

$\mathrm{E}$

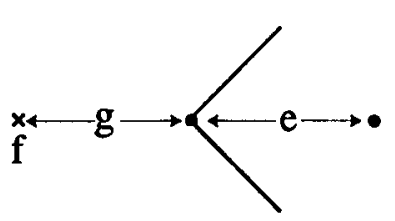

Figure 2. Panels A-D: Typical configurations of Experiment 1 . The configuration always contained a fin set (a) in which each fin was oriented at $45^{\circ}$ from the horizontal axis, a vertex dot (b), and a reference dot (c). Half of the configurations also contained a horizontal line segment (d) that passed through the vertex dot. The vertex dot was centered on the page, and the reference dot was placed at various distances to one side (not illustrated). Concave configurations are shown in panels $A$ and $D$; convex configurations are shown in panels $B$ and C. Variations of fin length are not illustrated. Panel $E$ : The subject was required to judge the span between the vertex and reference dots, which is described as the "stimulus span" (e), and to select and mark a point on the side of the page opposite the reference dot. This mark is described as the response dot (f), with the distance between the response and vertex dots being designated the response span (g). 
Shaft state. When the shaft was present, it consisted of a horizontal line segment that crossed the entire page, passing through the vertex and reference dots. When absent, the page contained only the dot pair and the fin set. A shaft is present in Figures $2 \mathrm{~A}$ and $2 \mathrm{C}$ and is absent for the configurations shown in Figures 2C and 2D.

Stimulus span. This factor (not illustrated) specified the distance to be judged and was manipulated by varying the position of the reference dot along the horizontal axis. The vertex dot and fins were always positioned at the center of the page.

Fin length. The length of each member of the fin set was set at one of the four values indicated above (variation of length not illustrated).

Side. This task condition consisted merely of whether the reference dot was located to the left or right of the centered vertex dot. For this experiment, the response would be given on the opposite side. Figure $2 \mathrm{E}$ shows the reference dot on the right, with the subject's response being given on the left.

Subjects and Test protocols. Eight undergraduates served as paid subjects. All were naive with respect to the phenomenon under investigation and were found to have normal $(20 / 20)$ visual acuity for each eye, some having the benefit of correction with contact lenses.

Each subject was provided with a test set, the sequence of pages in the set being in a different random order for each person. The subject evaluated the configurations with the aid of a viewing stand. The stand consisted of (1) a viewing/marking surface that was inclined $15^{\circ}$ toward the subject, relative to the plane of the table, (2) edge stops to aid in proper alignment of the page, and (3) a mask, similar to that used by Weintraub and Brown (1986), with eye slots that ensured a viewing distance of $46 \mathrm{~cm}$. The line of view from the mask was centered on the page and was orthogonal to the plane of the page.

The subjects were told that the stimulus material tested their ability to evaluate distance, and that they should transfer each page of the stimulus set to the surface of the stand, one at a time, taking care to align the page with the edge stops. Using the first few pages of the test set as examples, they were shown several alternative configurations that might be encountered. They were told that, for each of the pages, the task was to judge the distance between the central and lateral dot, registering their judgment by selecting and marking a location on the opposite side of the center. They were further instructed that if the stimulus displayed a horizontal line, they should place their mark on this line. If there was no line, they were told to mark the page along an imaginary horizontal axis line that crossed through the dots. They were told that if they were not satisfied with their initial judgment, they could erase and select a new location (an option that was seldom exercised)

When it was certain that the subjects understood the task demands, they were allowed to proceed through the stimulus set at their own pace; all completed the task in under $1 \mathrm{~h}$.

\section{Results}

Response span was measured to the nearest half millimeter, this value being compared with the distance between the stimulus dots to derive percent error for each of the configurations (i.e., response span minus stimulus span, divided by stimulus span). With this procedure, contraction of distance yields a value whose sign is negative, and, with expansion of distance, it is positive.

Some subjects appeared to have difficulty in following instructions for the no-fin control configurations. For this condition, the stimulus consisted of two dots, one that was centered on the page (nominally, the vertex dot) and the other lying to one side (the reference dot). They were instructed to judge the stimulus span and to designate an equivalent response span by placing a dot to the open side of the page (i.e., using the vertex dot as the starting point). At close distances, some subjects responded to the wrong side, either forgetting the instructions or not being able to discriminate which dot was centered on the page. This violated the counterbalance of the experiment and introduced the possibility that the size of the response might be partly due to eccentricity of the selected span. Nonetheless, this did not present much of a threat to the body of the experiment, since the concave and convex treatments serve as mutual control conditions in the interpretation of effect, and the no-fin condition would not have been used in the statistical analysis of the results in any event. Therefore, we have ignored the violations of instruction and scored the responses to the no-fin configurations by measuring the distance between the response dot and the nearest stimulus dot. Mean values across subjects indicate that there was mild expansion of span at short distances and that there was contraction at the greater distances. Specifically, the distortion was $11 \%, 3 \%,-3 \%$, and $-6 \%$ at $1-, 2-, 4-$, and $8-$ deg spans, respectively.

To facilitate comparison of concave and convex versions on the same scale, we have reversed the sign for all data for the concave condition. Thus, contraction of span for concave configurations and expansion of span for convex configurations are given as positive percent errors (except for in Figure 7, as explained subsequently).

Note that some factors were included in order to be thorough, with the expectation that one or more of these variables would have no differential effect on judgment. To determine whether this was the case, the data were submitted to preliminary evaluation using a five-way, withinsubjects analysis of variance (ANOVA). This analysis did not find significant differential effects as a function of side [whether the span to be judged was presented to the left or right; $F(1,7)=0.18$ ] or shaft state [presence or absence of the horizontal line segment; $F(1,7)=2.45]$. Additionally, there was no indication of interaction of these factors with other task conditions, so the data were collapsed across side and shaft state.

Resulting means were reanalyzed with a three-way, within-subjects ANOVA. We reasoned that any summary that collapsed the data across the two versions of the Müller-Lyer would not provide a meaningful result, so only the analyses that retained version as a factor were calculated. The main effect for version (concave vs. convex) was not significant $[F(1,7)=1.48]$. However, the two-way interaction of version $\times$ stimulus span was significant $[F(3,21)=30.15, p<.001]$, as was version $\times$ fin length $[F(3,21)=4.71, p<.05]$. The three-way interaction of version $\times$ stimulus span $\times$ fin length also was significant $[F(9,63)=3.00, p<.01]$.

The means for these treatment conditions are shown in Figures 3 and 4 . Figure $3 \mathrm{~A}$ shows the version $\times$ fin length interaction. There is clearly a differential of effect for the two versions, with the convex configuration providing a fairly constant level of expansion of the span to be judged and with the concave configuration yielding a contrac- 
A

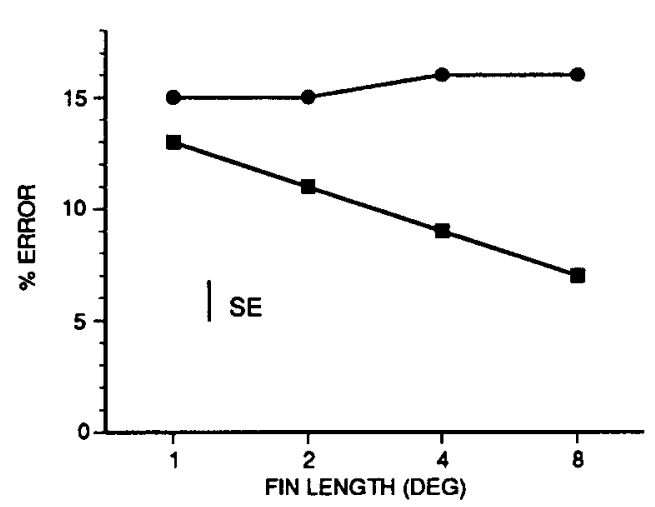

B

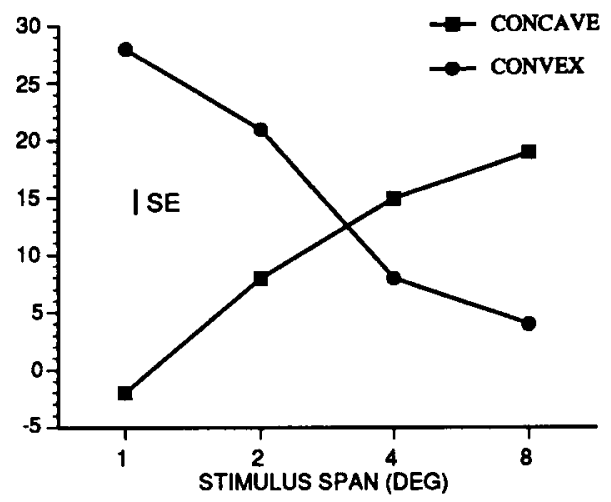

Figure 3. (A) In Experiment 1, convex configurations produced high error levels at all fin lengths. For concave configurations, short fin length produced high error levels, but the strength of effect declined as fin length was increased. (B) A differential pattern was found for the two configurations as a function of the span to be judged. For convex configurations, error levels declined as a function of increasing stimulus span; for concave configurations, they increased. Note that, in this figure and in subsequent data figures, positive error indicates contraction of the stimulus span for concave configurations and expansion of the span for convex. Variance was very similar irrespective of the treatment conditions, so each panel shows average standard error as an inset.

tion of span that declined as a function of fin length. This differential pattern of effect supports the argument that the two versions do not have symmetrical effects on judgment, as will be discussed more extensively below.

Figure 3B shows the interaction of version $X$ stimulus span. A differential of effect is even more conspicuous here, with the strength of effect increasing for the concave version and decreasing for the convex version, as the span to be judged grows larger. In other words, for the concave configuration, the least distortion of perceived distance was found when the span was short, and the greatest distortion occurred at the longest span (i.e., at $8 \mathrm{deg}$ ). The reverse pattern was found with the convex version; with increasing distance, the subjects judged the span with greater accuracy.

In examining the data shown in Figure 3, and for all subsequent statistical analysis, it should be remembered that the sign of the error has been reversed for the concave data. This means that the positive percent-error values shown on the ordinate reflect contraction of distance for concave configurations and expansion for convex configurations. Thus, the overall direction of effect for each version is consistent with what one would expect with traditional Müller-Lyer configurations, and the statistical analysis assumes this differential influence on judgment. Significance is not claimed unless the overall strength of effect or functional relationship among the variables is dissimilar in other ways. Here, we find differences with respect to both fin length and the span to be judged.

The four panels of Figure 4 show the interaction of version $\times$ fin length $\times$ stimulus span. Each of the panels shows an increase of effect for the concave version and a decrease with the convex version, as the span to be judged is increased. The patterns differ somewhat across the four fin-length conditions, providing the basis for a significant three-way interaction, as reported above. However, we are more impressed by the similarity of effects across the four panels than with the differences. The data suggest a differential for concave and convex configurations that was not previously suspected - namely, that the former have their greatest influence on perceived distance when the spans to be judged are large, whereas the latter produce more distortion when they are short.

\section{EXPERIMENT 2}

In Experiment 1, we found an unexpected relationship between the two versions of the Müller-Lyer with respect to the distance being evaluated. We conducted Experiment 2 partly to be certain that these effects were reliable and to see how fin angle might influence the results. Thus, subjects were asked to judge the span between the vertex and reference dots in the presence of the following factors: (1) version (concave/convex), (2) stimulus span, (3) fin length, and (4) the orientation of fins (i.e., fin angle). Having adopted nonangular terms for describing the two versions of the Müller-Lyer, we were free to use a more convenient method for specifying fin angle. Specifically, fin angle was measured as the smallest departure from the judgment axis (i.e., the acute angle). This convention facilitates comparison of data for "symmetry of effect" at corresponding angles.

In Experiment 1, the presence of the shaft segment was not found to produce a significant differential of effect; therefore, it was not included here or in the subsequent experiments. To keep the permutations of treatments to a manageable level, we used two, rather than four, fin lengths. Each combination of the stimulus treatments was presented with the reference dot to the left or 

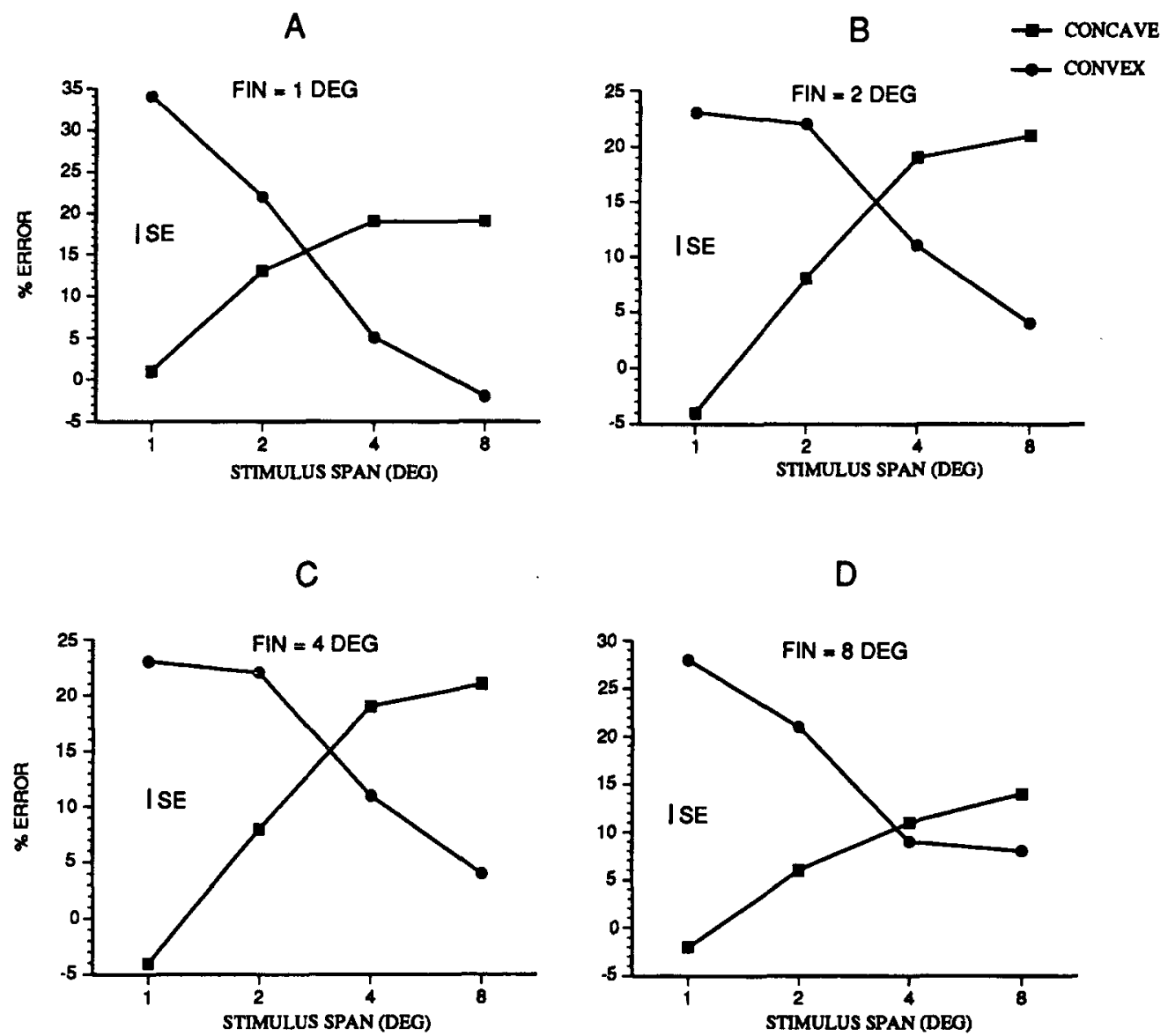

Figure 4. The three-way interaction of the major task variables of Experiment 1 is shown in four panels. Although the interaction was significant, the functional relations suggested in Figure 3B appear as a consistent feature of the plots irrespective of fin length.

right of center to prevent the development of response bias. However, the data were collapsed over this covert variable, since, in Experiment 1, we had not found any sign of differential effect.

\section{Method}

Stimulus materials and Procedure. Unless otherwise stated, all stimulus and test conditions were the same as those in Experiment 1 . The treatment conditions consisted of two levels of version (concave, convex), four levels of stimulus span $(1,2,4$, or $8 \mathrm{deg}$ ), two levels of fin length ( 2 or $8 \mathrm{deg}$ ), and five levels of fin angle $\left(15^{\circ}-75^{\circ}\right)$. Permutations of these conditions (with two levels of side as a covert variable) provided 160 pages of test material. Additionally, a set of pages was created in which each of the fins was oriented at $90^{\circ}$. Here, the concept of concave or convex is moot, and scores from this material were not included in any statistical analysis for the differential effect of version. However, the response to the $90^{\circ}$ configurations is included in the plot of fin-angle effects and provides a useful bridge for showing the transition from concave to convex versions of the Müller-Lyer. The $90^{\circ}$ subset added 16 pages, providing a total test set of 176 pages. We feared that size of the experimental set would press the limit of vigilance, so it was decided that a replication of the no-fin control condition was not needed.

Subjects. Eight new subjects were tested in Experiment 2, each being provided with a different random order of the test pages. The subjects were required to take a brief rest break $(5 \mathrm{~min})$ after half of the material had been judged. Even with the large set size and with a break, all finished in under $1 \mathrm{~h}$.

\section{Results}

The data were evaluated with a four-way, withinsubjects ANOVA, again calculating statistical values only when version was retained as a factor. The main effect for version was significant $[F(1,7)=30.98, p<.001]$. Each of the two-way interactions involving version were also significant, as follows: version $\times$ stimulus span $[F(3,21)=$ $123.05, p<.001]$, version $\times$ fin length $[F(1,7)=12.25$, $p<.01]$, and version $\times$ fin angle $[F(4,28)=8.44, p<$ $.001]$. The three-way interaction of version $\times$ fin length $\times$ fin angle was not significant $[F(4,28)=0.80]$. However, the other three-way interactions were significant, as follows: version $\times$ stimulus span $\times$ fin length $[F(3,21)=$ $3.44, p<.05$ ] and version $\times$ stimulus span $\times$ fin angle $[F(12,84)=4.11, p<.001]$. Finally, the four-way interaction was not significant $[F(12,84)=1.30]$.

The means for Experiment 2 are plotted in Figures 5-7. The first two panels of Figure 5 show that there is a clear differential in the absolute level of effect for the two Müller-Lyer configurations, as confirmed by the sig- 
nificant main effect for version, reported above. In Experiment 1 , these effects (averaged across all other conditions) differed by $5 \%$, which was not sufficiently large to produce a significant main effect. In Experiment 2, the differential in average effect was $7 \%$, yet this proved to be significant at $p<.001$. The difference between the two experiments was likely due to the particular combination of variables that was used. Experiment 1 included a 1-deg fin length, which generated perceptual distortions that were in the same range for both versions of the MüllerLyer (see Figure 3A). Elimination of this fin length in Experiment 2 had the effect of reducing overlap in the data. Additionally, fin angle produced a substantial differential of effect for the two configurations (see Figure 5B), and this also contributed to a significant main effect for version.

The significant interaction of version $\times$ fin length is shown in Figure 5A. It is clear that the pattern is the same as was found in Experiment 1-namely, that the convex configuration produced a relatively constant level or error irrespective of fin length, but, for the concave configuration, the error levels declined as the fins were increased in length.

Figure 5B shows the effect of fin angle for the two stimulus configurations. Both versions produced a biphasic pattern, peaking at $30^{\circ}$ for the convex stimulus and in the $45^{\circ}-60^{\circ}$ range for the concave stimulus. Thus, a convex fin set had the greatest influence on perceived span when the angle between the fins was relatively small, and that influence declined as the fins were spread. For the concave fin set, however, the influence on judgment was relatively small for the most acute and least acute configurations and was maximal when the relative angle between the fins was intermediate. These differential effects for fin angle will be considered again when Figure 7 is evaluated.

The interaction of version $\times$ stimulus span is presented in Figure 5C. It is clear that the pattern of effect seen in Experiment 1 has been replicated. The convex configuration produced the largest errors when the span
A

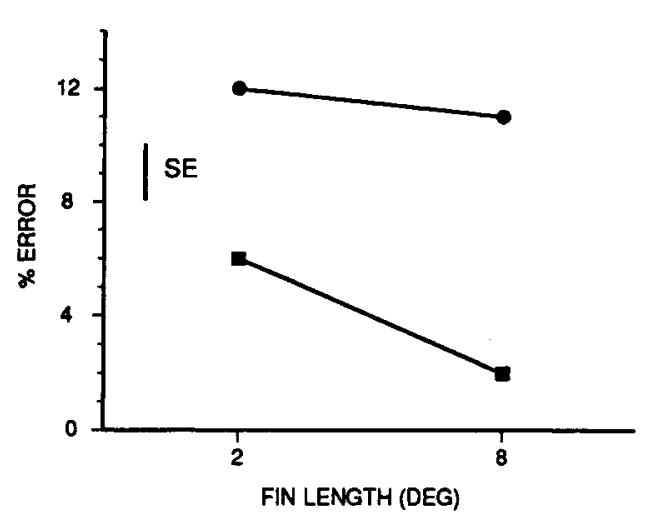

B

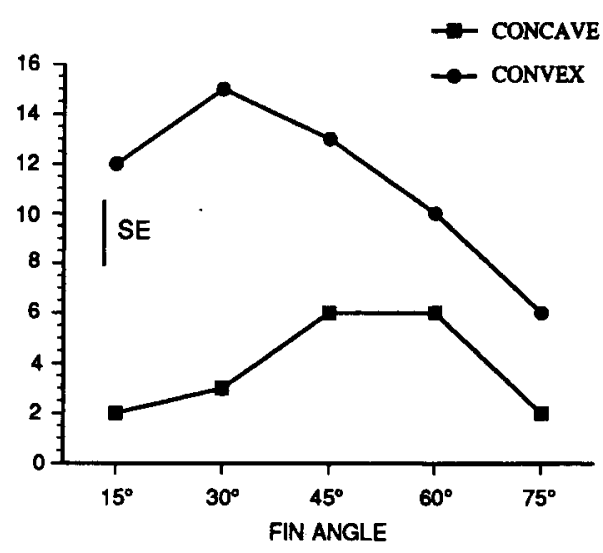

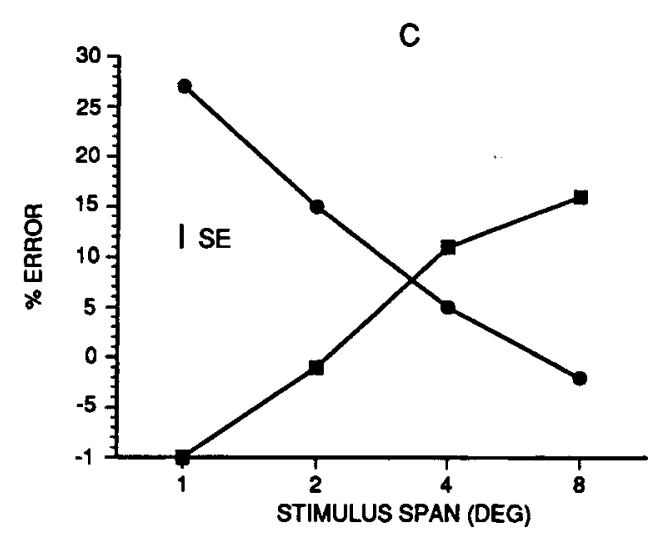

Figure 5. (A) The results of Experiment 2 appear to confirm a differential effect of fin length for concave and convex versions. Error levels are relatively high and constant for convex configurations but decline as a function of increasing length for concave configurations. (B) Manipulation of the angle of the fin set produced differential effects for the two versions of the Müller-Lyer. (C) Confirming the results of Experiment 1, the two versions are opposite in relation to the span to be judged. For convex configurations, the error level is a decreasing function of stimulus span; for concave configurations, the strength of effect increases as the span grows larger. 
A

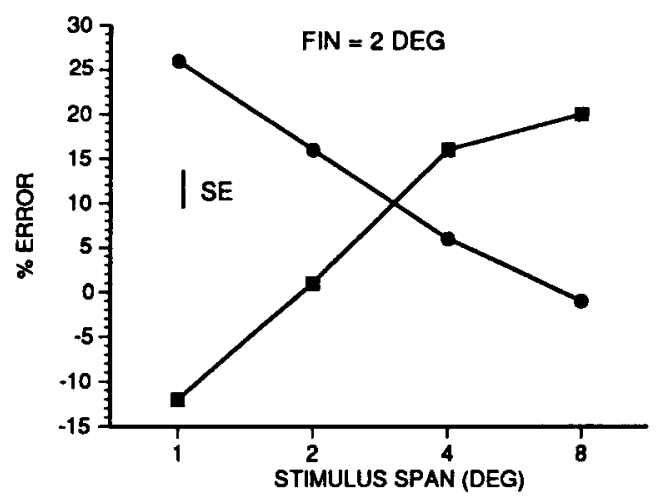

B

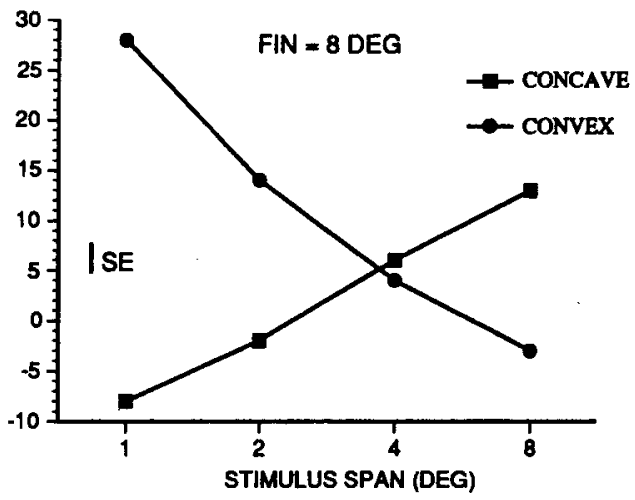

Figure 6. In Experiment 2, a differential functional relationship of version to stimulus span can be seen at each of the tested fin lengths, similar to what was found in Experiment 1 (Figure 4 ).

to be judged was short, and this effect declined as the span was increased. A reversed functional relationship was found for judgments of the concave configuration.

This differential effect for the two versions can be seen by plotting the data for the three-way interaction: version $\times$ stimulus span $\times$ fin length (shown in Figure 6 as two panels). As in Experiment 1, for each fin length, the convex configuration produced a declining effect as stimulus span was increased, and the reverse was true for the concave configuration.

Figure 7 shows the version $\times$ stimulus span $\times$ fin angle interaction. Here, we have folded out the angles in the two versions, placing the concave set on the left and the convex set on the right of the figure. The angles are ordered such that, for each, the smallest angle lies at opposite ends of the series, and we have inserted the means for the $90^{\circ}$ configurations in the middle. Thus, one can conceive of the positions on the abscissa as a progression from the most concave configuration (at the far left) to the most convex configuration (at the far right). In other words, considering the span that lies between the vertex dot and the reference dot, the values on the far left come from a configuration in which the fin segments lie in greatest proximity to that space, and those on the far right have the least proximity. With this method of plotting, the $90^{\circ}$ position essentially becomes the zero of a Cartesian coordinate system, with the concave angles lying to the negative side. To see functional relationships, the concave data should not be (and are not) reversed in sign.

Each stimulus span is given a distinguishing symbol, and these are connected to better show the pattern of effects. The trends are predominantly monotonic as one moves from the smallest concave to the smallest convex angles; curvilinear components appear to be present, however, especially for short stimulus spans.

The plot of effects in Figure 7 should provide some caution in the interpretation of what is shown in Figure 5B. The latter graph shows means that have been averaged across stimulus span. If the biphasic patterns of Figure 5B were reflected in each of the curves of Figure 7, one might be confident in concluding that the averages provide a useful index of the underlying distortion mechanism. However, this does not appear to be the case. For the concave configurations, a biphasic function with a peak in the $45^{\circ}-$ $60^{\circ}$ range appears to be present for short spans, but not for those that are $4 \mathrm{deg}$ or more. On the convex side, the overall trend is for an increase in effect as the angle becomes smaller, but the peak effect seen at $30^{\circ}$ in Figure 5B is not a consistent trend for each stimulus span. At the very least, this result precludes a firm conclusion about the role of fin angle and suggests that analysis and discussion of this factor should be done in relation to the differential effects of the span being judged.

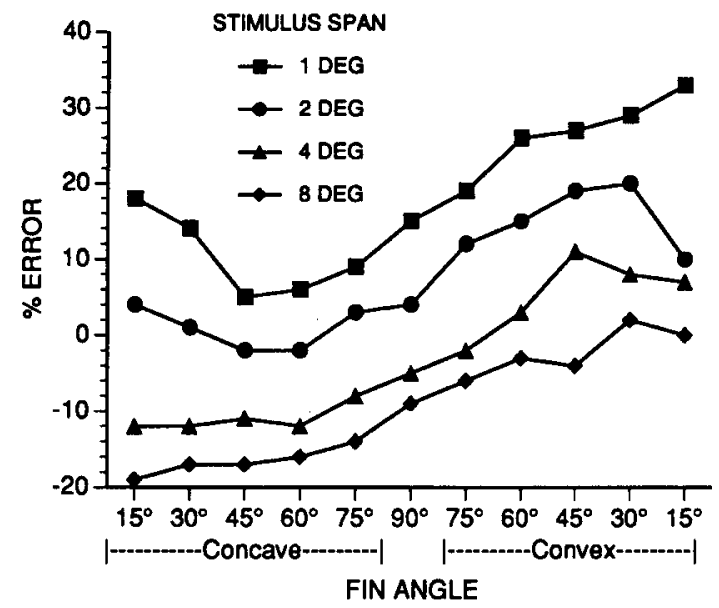

Figure 7. The three-way interaction involving fin angle (Experiment 2 ) is shown by plotting the data from concave configurations on the left and from convex configurations on the right. A consistent pattern is evident, with a clear differential for the two versions as a function of the stimulus span being judged. Manipulation of angle produced a significant differential of effect. 


\section{EXPERIMENT 3}

The test protocols used in Experiments 1 and 2 provided useful information, the most important being to show a strong differential in effect as a function of the span to be judged. It should be recognized, however, that requiring judgment of span on one side of the fin set and then placing the response on the other introduces some ambiguity. Is the fin set acting on the space being evaluated (the stimulus span), the space being used to render the judgment (the response span), or both? One might reasonably expect that it would act on both sides, requiring any distortion of the perceived stimulus span to be transferred into a space that was distorted in the opposite direction. However, precise specification of this hypothesis becomes difficult if one also factors in the effects of distance. Experiments 1 and 2 showed that the net error level is an increasing function of distance when the reference dot lies on the concave side and is a decreasing function of distance when it lies on the convex side of the fin set. It is not clear how one would see this differential pattern if the fin set produces simultaneous distortion on the convex and concave sides of the fin set.

To help clarify the issue, Experiment 3 was conducted, requiring that the subject's response be placed away from the fin set. In other words, the subjects selected and marked a point as a displacement from the reference dot. This does not provide an absolute guarantee that the space being used for the response is immune from the influence of the fin set. Experiments 1 and 2 showed effects with spans up to $8 \mathrm{deg}$ - whether the effects could extend even farther is unknown. Nonetheless, one would anticipate that, under these conditions, the net error would reflect a single direction of bias (concave or convex) rather than a combination of the two.

In fact, we tested two different response protocols, identified as Experiments $3 \mathrm{~A}$ and 3B. In Experiment 3A, the subject indicated the judgment of stimulus span by responding in the open space beside the reference dot. This is described as the external procedure, in that the response dot was placed to the far side of the reference dot (i.e., away from the fin set). In Experiment 3B, the subject divided the space between the reference and vertex dots. This method is described as requiring an internal procedure, in that the response dot was placed between the reference and vertex dots. We used the two alternative procedures to determine whether a judgment of symmetrical distance within a span is affected differently than a judgment that requires transfer of a perceived span. Comparison of the two methods required an adjustment of the stimulus span convention, as described below.

Thus, for each of the response modes, the following treatment factors were manipulated: (1) version, (2) stimulus span, and ( 3 ) fin length. The two response modes of Experiments $3 \mathrm{~A}$ and $3 \mathrm{~B}$ were tested in separate sessions using the same subjects.

\section{Method}

Stimulus materials and Procedure. Unless otherwise specified, stimulus materials and test protocols were identical to those used previously. The treatment conditions consisted of two levels of version (concave/convex), four levels of stimulus span $(1,2,4$, and $8 \mathrm{deg}$ ), four levels of fin length $(1,2,4$, and $8 \mathrm{deg})$, and two levels of side (as a covert variable). Each permutation was tested twice; thus, the test set for each response mode contained 128 pages.

To provide for comparability of distances for the two experiments ( $3 \mathrm{~A}$ and $3 \mathrm{~B}$ ), the reference dot for internal configurations was placed at twice the distance of the nominal levels given above. To be specific, for the 1-deg configuration, the reference dot actually lay at $2 \mathrm{deg}$; for the 2-deg configuration, it was placed at $4 \mathrm{deg}$, and so on. Thus, when a subject was asked to divide the space for an 8-deg configuration, in fact, the reference dot was positioned at $16 \mathrm{deg}$. For a veridical judgment, the subject would respond at $8 \mathrm{deg}$. This provides for comparability to the demands of the external responsemode procedure, wherein the subject is asked to judge a reference dot located at 8 deg from the vertex and responds veridically by selecting a point at $16 \mathrm{deg}$. If the reader is confused by this convention, try reading the four levels of stimulus span as "veridical response span," since, in the absence of error, the subject will place the response dot at $1,2,4$, or $8 \mathrm{deg}$ from the reference dot, irrespective of the response mode.

The internal response mode also required a new operational definition of what spans are judged, because the subject is not matching the distance between the vertex and reference dots. When asked to divide the space between the two stimulus dots, the subject chooses a location in which the span between the vertex dot and the response dot are judged to be the same as the span between the response dot and the reference dot. Thus, for this task, we define the former as the stimulus span and the latter as the response span. The accuracy of this judgment is measured as the distance between the reference and response dots; therefore, if the fin set causes contraction of the stimulus span, the subject will select a point that is too close to the reference dot.

For the stimulus material of both experiments, to allow for point positions that could span up to $16 \mathrm{deg}$, it was necessary to move the fin set 6 deg off of center. The fin set was moved toward the side opposite of the reference dot, thus providing a majority of the page space for placement of the reference and response dots.

Subjects. Eight new subjects judged the subset of material for each response mode (i.e., external vs. internal placement of the response dot) in two sessions. The order of pages for each subset was different for each subject.

\section{Results}

The data for response to the left or right side of the page was averaged, and this provided the raw scores for statistical analyses. The values in each experiment were evaluated with a three-way, within-subjects ANOVA.

For Experiment $3 \mathrm{~A}$ (requiring an external response mode), the version treatment (concave/convex) produced a significant differential effect $[F(1,7)=8.47, p<.05]$. The version $\times$ stimulus span interaction was significant, but the version $\times$ fin length interaction was not $[F(3,21)=$ $27.05, p<.001$, and $F(3,21)=2.28$, respectively]. The three-way interaction (version $\times$ stimulus span $\times$ fin length) was significant $[F(9,63)=3.55, p<.01]$.

For Experiment 3B (requiring an internal response mode), the main effect for version was also significant $[F(1,7)=8.43, p<.05]$. Comparable effects were found 


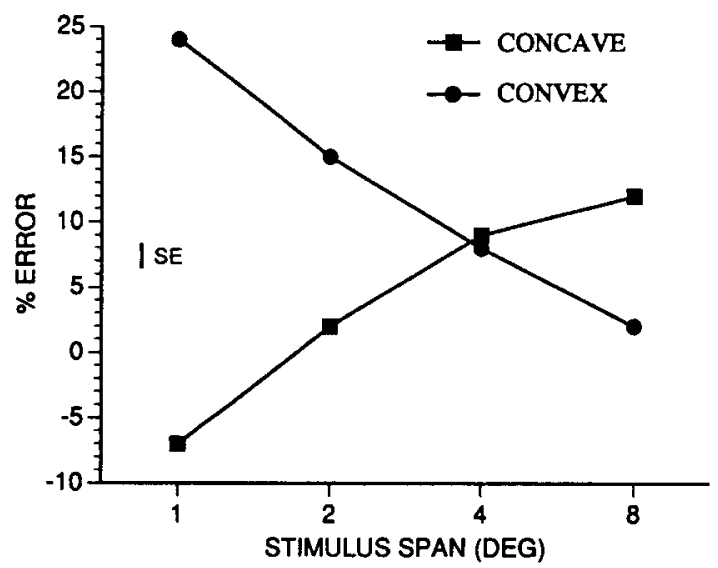

Figure 8. The major variables of Experiment $3 A$ were the same as before, but the response span was indicated as distance from the fin set to the opposite side of the reference dot (see Method section for clarification). The stimulus span differentials for the two versions were still evident, indicating that the effects seen in Experiment 1 were not an artifact of the judgment being rendered in the zone that is adjacent to the fin set.

with the interactions as well, with the version $\times$ stimulus span interaction being significant and with version $\times$ fin length not showing a differential $[F(3,21)=8.20, p<$ .001 , and $F(3,21)=2.00$, respectively]. For the internal response mode, the three-way interaction was not significant $[F(9,63)=1.50]$.

The differential effects of the two versions as a function of stimulus span for Experiment 3A are plotted in Figure 8 . The pattern appears to be essentially the same as was found in the earlier experiments, with the concave version producing increasing errors as a function of the span to be judged, and the convex version yielding a decline in error level. The two functions appear to cross slightly farther to the right (see Figures $2 \mathrm{~B}$ and $4 \mathrm{C}$ for comparison). The four panels of Figure 9 show the threeway interaction, and it seems clear that this shift becomes larger as fin length is increased. However, it is not known whether this is a reliable effect, nor what might be the significance of such a finding.

The effects for the internal response mode (Experiment 3B) are plotted in Figure 10. Distortion effects for the concave and convex versions were in the same general direction as found previously, with percent errors increasing as a function of distance for concave configurations and decreasing for convex. However, overall, the size of effects was considerably weaker, and the point of intersection is shifted even farther to the right. The threeway interaction of version $\times$ stimulus span $\times$ fin length was not significant, and, therefore, one can take the effects shown in Figure 10 as representative for each fin length.

\section{FUNCTIONAL MODELING}

A major goal of the present research was to determine whether the distortions that are produced with the clas- sic Müller-Lyer configurations can be seen using a single fin set. We have, indeed, found contraction when the stimulus span lies to the concave side of a fin set and found expansion when it lies on the convex side. However, we also found that the strength of effect is substantially influenced by the span being judged and that the functional relationship is opposite for concave and convex configurations. Given this fact, it would be useful to give additional attention to the role of distance. Deriving an algebraic function for the effects would be especially desirable.

For statistical purposes, we adopted a standard method for expressing the magnitude of metric distortionnamely, percent error. This was appropriate because this index has been used in most previous studies of the Müller-Lyer effect, and also because it seemed to be relatively "theory neutral." Calculating the value as response span $\left(S^{\prime}\right)$ minus stimulus span $(S)$ divided by stimulus span is equivalent to $S^{\prime}=(\%$ error +1$) S$, which is formally the same as $S_{i}^{\prime}=a_{i} S_{i}$. Thus, for a given value of $S$, there is a corresponding value of the $a$ parameter that may change in a linear or nonlinear manner.

It should be clear, however, that this method of expressing the effect is not optimal from an algebraic standpoint and has the potential for obscuring the true quantitative relationships. For example, if the span were being measured from the wrong location, the system that evaluates distance would simply add or subtract a constant value from $S$, that value being the distance between the true location of the dot and the point from which perceptual measurement began. In this case, it would be best to express the functional relationship as $S^{\prime}=S \pm c$. While the addition of a constant quantity can be specified as a progression of values of a multiplier (i.e., the parameter $a_{i}$ ), it is obvious that this is not the best index of the functional relationship.

For purposes of functional modeling, therefore, we have returned to the raw data in search of a simple method for summarizing the relationship between stimulus and response spans. The most basic question was whether the differential effect of the concave and convex versions might be given as a simple algebraic function. Because we saw approximately linear changes in percent error as octave steps of distance, we thought it likely that some form of geometric progression would be needed to relate the raw values of $S$ and $S^{\prime}$. However, in an initial plot of the data from Experiment 1, a simple linear relationship between the variables was found (see Figure 11). In each experiment, the data points for concave and convex versions fell along straight lines.

To provide a more comprehensive analysis of this finding, we used a least squares regression program to evaluate the data from the four experiments. The program calculated optimal coefficients and degree of fit for a linear equation relating $S^{\prime}$ to $S$, specifically, $S^{\prime}=a S+$ $b$. Best fits were determined for the summary means of the version $\times$ stimulus span interaction, the data having been collapsed across other treatment conditions. Additionally, the degree of fit for the no-fin control condition was examined. 
A

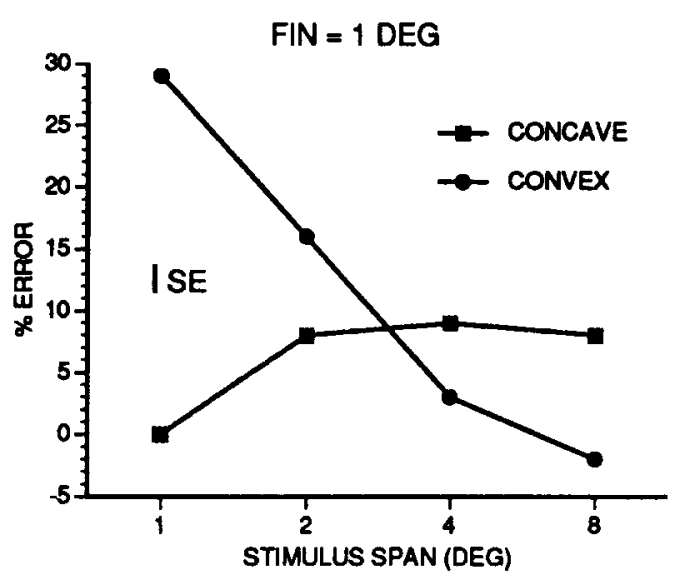

C

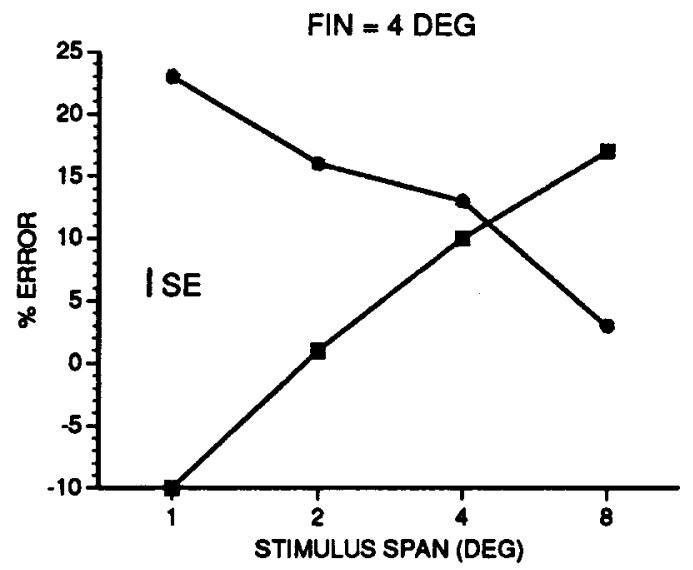

B

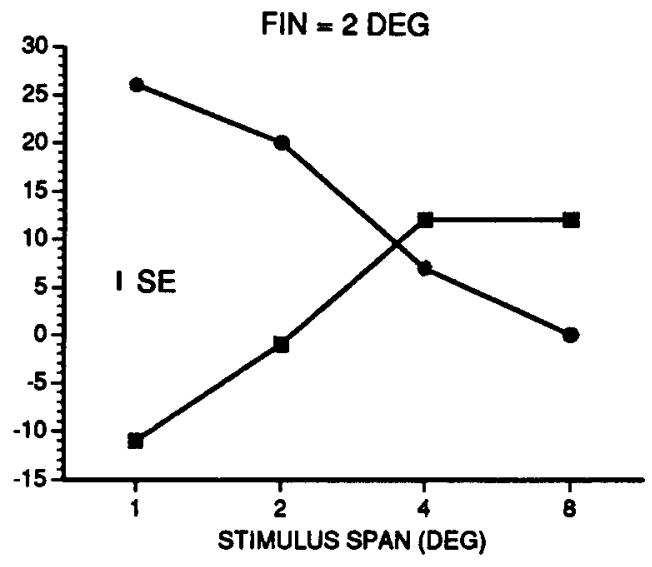

D

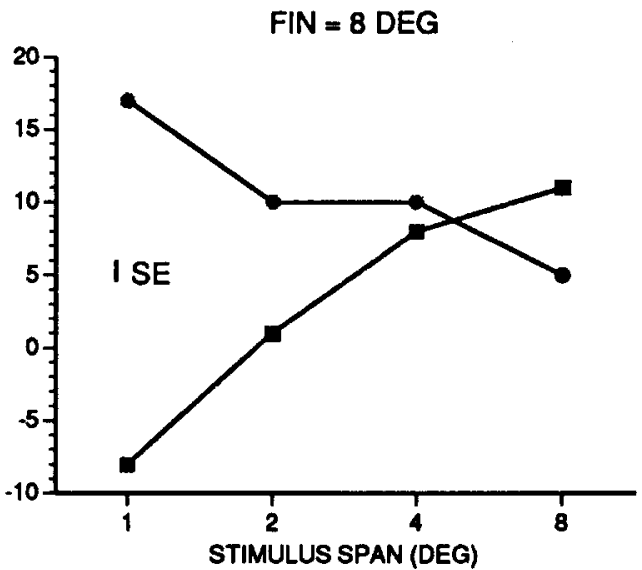

Figure 9. The three-way interaction of Experiment $3 \mathrm{~A}$ was significant, and one can see differences in effect as a function of fin length. Nonetheless, the dominant characteristic of the plots is an opposite function for each version in relation to the stimulus span being judged.

Table 1 shows the results of the least squares calculations, providing the values of the two coefficients in each experiment, as well as the index of fit $\left(R^{2}\right)$ of the data to the model. It can be seen that each set of data had an index of fit of $99 \%$ or better. Indeed, fitting the data at each fin length for Experiments 1, 2, and 3 and at each fin angle in Experiment 2 provided $R^{2}$ values in this same range (the smallest being .9894). It seems clear that the best quantitative summary of experimental effect is provided by a linear equation in which one parameter $(a)$ adjusts the perceived span as a proportion of the true stimulus span, and another parameter (b) adds a constant amount to the perception.

The four panels of Figure 11 show the plot of $S$ and $S^{\prime}$ means for the four experiments, as well as the regression lines, using a linear scale for the ordinate and abscissa. For Experiments 1, 2, and 3A, the slopes and intercepts are quite similar. For Experiment 3B, the intercepts for each version were almost zero, and there was only a small differential in slope. Nonetheless, statistical differences for these means would be the same as for comparisons in which the scores were expressed as percent error. Thus, even for Experiment 3B, the differentials in slope for the concave and convex versions would be significant.

As indicated above, there was a linear relation between $S$ and $S^{\prime}$ at each level of fin length and fin angle. However, there would be no benefit in presenting these plots, since they are almost identical to what is shown in Figure 11. Instead, to better delineate the effects of these treatments, we have extracted the values of the $a$ and $b$ parameters at each level of fin length and angle.

To determine the influence of fin length on the fitted parameters, we examined the data from Experiments 1 and $3 \mathrm{~A}$ (Experiment 2 used only two values of length, and this factor was not significant in Experiment $3 \mathrm{~B}$ ). The change in $a$ is shown in the first two panels of Figure 12 , and the change in $b$ is shown in the second two panels. There appears to be an asymmetry of influence with respect to the concave and convex versions. For both experiments, the a parameter rises for convex and drops 


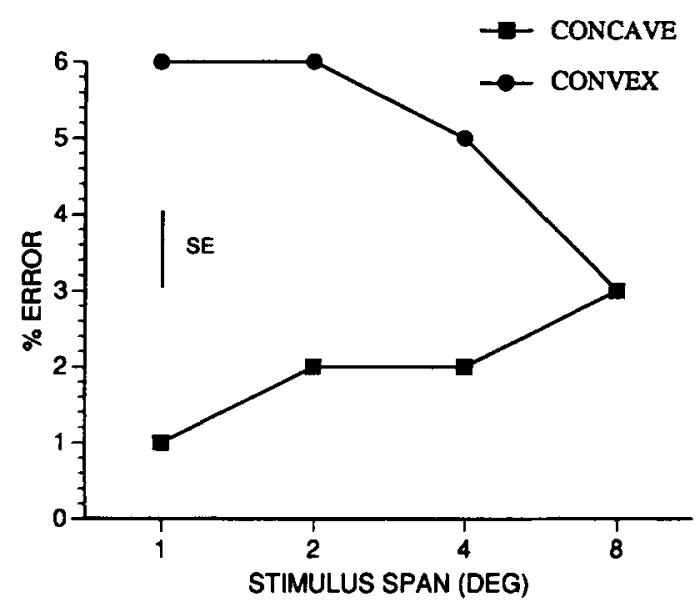

Figure 10. In Experiment 3B, the subject was asked to divide the space between the vertex and reference dots. Although the version $\times$ stimulus span interaction (shown here) was significant, the overall levels of error were substantially lower than those found in Experiments 1, 2, and 3A. Additionally, the intercept was shifted farther to the right, and fin length did not produce a significant differential of effect.

for concave as the length of the fin set increases from 1 to $2 \mathrm{deg}$. Thereafter, the value of this parameter rises gradually with increasing length for both versions. Corresponding and nonsymmetric curves are also found for the $b$ parameter.

If one were considering only Experiment 1, it would be tempting to speculate that an underlying effect contained a hidden symmetry for the two versions. Looking at Figure $12 \mathrm{~A}$, one might note that the curves would be fairly symmetric if the value of $a$ for the concave version were lowered slightly at $4 \mathrm{deg}$ and somewhat more at $8 \mathrm{deg}$. One might suspect that small variations in the data had resulted in the "optimal" regression line being slightly off the mark. Indeed, if the discrepant values of the a parameter were modified, essentially forcing the convex and concave curves to be symmetric, then recalculation of optimal fit to the data would result in a rise of the corresponding $b$ values. The resulting curves for both parameters would then be fairly symmetrical with respect to the concave and convex versions, and the level of fit would still be in the $99 \%$ range.

The results of Experiment $3 \mathrm{~A}$, however, appear to provide a replication of the asymmetry. Given this result, we are reluctant to force any change in the extracted values.

Figure 13 plots the values of $a$ and $b$ that were extracted from the data of Experiment 2 (i.e., derived from the means at each level of fin angle and collapsing across fin length). The $a$ values (Figure 13A) appear to change as a linear function of angle, being smallest at the most extreme concave angle and being largest at the more extreme convex angles. A regression line has been fitted to the data.

The changes in the $b$ parameter as a function of fin angle are shown in Figure 13B. The plot does indicate a systematic change in the values of $b$, but the rather amor- phous shape of the curve does not suggest a simple functional relationship.

As outlined above, one would expect some variability in the values of extracted parameters. The $a$ and $b$ values at each level of fin angle are based on an optimal fit to four means (i.e., the means at each stimulus span), similar to the plots in Figure 11. For any given set of means, one determines the slope (a) and intercept (b) of the bestfitting regression line, and the particular values for the pair may be off slightly due to chance variability among the four means from which they are derived.

We think it likely, therefore, that the true values of the a parameter will fall on the regression line that is shown in Figure 13A, with the departures from this line being due to chance. If so, then one might ask what would be the value of the corresponding $b$ parameter if each $a$ was forced to the regression line. To answer this question, we recalculated the best-fitting regression (across the four stimulus spans for each level of fin angle) but requiring that each $a$ be at the level predicted by the regression line shown in Figure 13C. With the value of $a$ being thus specified, the fit must be optimized by adjustment of the $b$ value. Figure 13D provides the new estimates for $b$. Now, the overall curve that connects the points is suggestive of a sinusoidal oscillation.

It should be noted that the values of the $b$ parameter lie in a very small range (i.e., between 0.30 and $0.55 \mathrm{deg}$ for the unadjusted estimates [Figure 13B] and not much different for the adjusted estimates [Figure 13D]). Thus, the variation from the lowest intercept to the highest is only about $0.25 \mathrm{deg}$ of visual angle. In the overall scale of judgment, this is extremely small, as can be seen by examining similar intercept values in Figure 11.

Additionally, it should be noted that adjustment of the a parameters does not greatly affect the fit of the model to the data. The regressions that provided the "forced" parameter values (shown in Figures $13 \mathrm{C}$ and 13D) had an index of fit $\left(R^{2}\right)$ of .9994 or better at each level of fin angle.

Some limited discussion of prior work may be useful at this point. As reviewed previously, most investigators have reported that the size of distortion changes as a function of fin angle, but they have not agreed on the shape of the function. Some have reported near linear effects as the angle was varied (e.g., Heymans, 1896; Pressey, 1974; Pressey \& Martin, 1990). It has been reported that fin length influences whether or not the effects are monotonic (e.g., Coren, 1986; Day \& Dickinson, 1976; Erlebacher \& Sekuler, 1969) and that the shape of the curve differs for the two versions (e.g., Goryo et al., 1984; Sekuler \& Erlebacher, 1971). Given our present modeling results, it is not surprising that diverse effects would be seen. Any index of distortion that is provided by a single parameter, such as percent error $\left(S_{i}^{\prime}=a_{i} S_{i}\right)$, cannot provide an adequate summary of treatment effects. The results from Experiment 2 do suggest that the a parameter changes as a linear function of angle, but then one must add the complex changes in $b$ to model the effects for a given configuration. For studies that have reported a linear change in percent error with changes in angle, one 

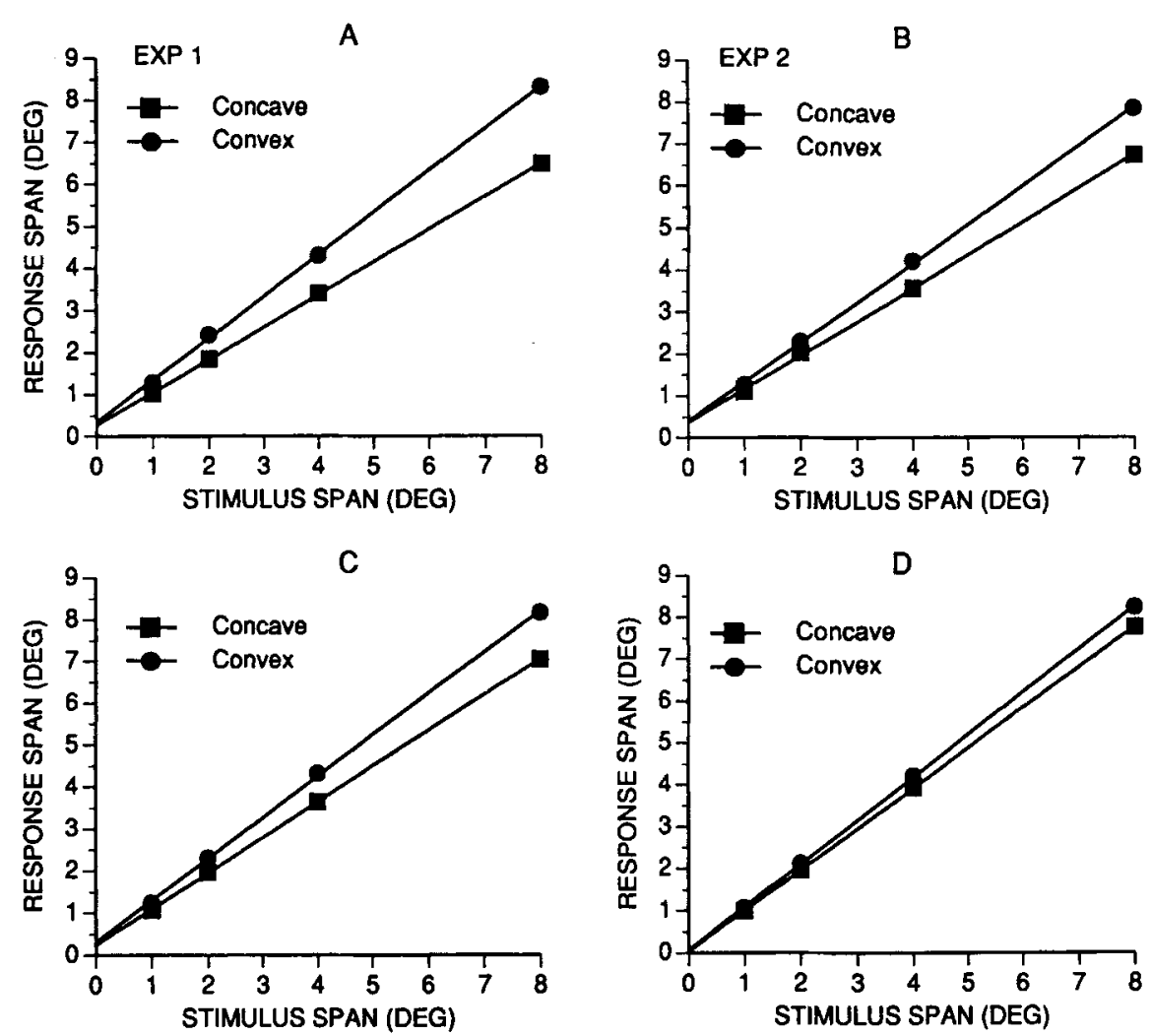

Figure 11. To better determine the functional relationship between the variables, response span $\left(S^{\prime}\right)$ was plotted against stimulus span $(S)$, using linear scales and not using percent error as the index of effect. For each of the four experiments, mean response span for the concave and convex versions (collapsed across other treatment variables) was found to be an almost perfect fit to the equation: $S^{\prime}=a S+b$.

might suspect that the specific test procedures caused the $b$ value to be near zero, as was the case here for Experiment 3B.

The primary and most certain conclusion that can be drawn from the formal modeling is that essentially all of the influence from a single fin set can be fitted using a linear function of the form $S^{\prime}=a S+b$. This equation matches the pattern of data across four octaves of stimulus span, with variation of fin length across four octaves and with variation of fin angle across essentially the full range of relative orientation. The equation even matches the control condition in which fins are not present.

Furthermore, it seems likely that we are dealing with a linear system in which the values of the two parameters are independent of one another. This conclusion is based on the differential patterns of effect shown in Figure 12, and more so in Figure 13. It would be difficult to conceive of a functional relationship between the values of $a$ and $b$ at the various levels of fin angle without adding an arbitrary geometric function (such as a sine wave). Yet the combination of $a$ and $b$ values at each fin angle predicts the distortion of perceived distance with the index of fit being $99 \%$ or better. A more parsimonious conclusion is that there are two independent parameters of perceptual distortion, one that multiplies the span being evaluated and one that is added to the resulting product.

\section{DISCUSSION}

While this is not the appropriate forum for a thorough review of the Müller-Lyer literature, we should provide a general overview of the major theories in relation to the present findings. Any brief summary runs the risk of blurring distinctions that each theorist would consider to be important. However, for present purposes, it is most efficient to group the explanations that have common characteristics.

Before proceeding, we need to clarify three points regarding terminology. First, we will continue to describe the two versions of the Müller-Lyer as concave and convex. These terms therefore will be substituted for the many others that have been used to describe the versions (e.g., fins-in vs. fins-out, acute vs. obtuse; or containing heads vs. tails, respectively). Second, in most of the previous work, the configurations being tested have contained a shaft. To facilitate comparison with the present experimental results, we will preferentially use the term span to describe the distances that are judged, whether or 
Table 1

Values for Parameters $a$ and $b$ and for $R^{2}$ for Experiments 1, 2, 3A, and 3B

\begin{tabular}{|c|c|c|c|}
\hline Version & $a$ & $b$ & $R^{2}$ of Fit \\
\hline \multicolumn{4}{|c|}{ Experiment 1} \\
\hline Concave & 0.7780 & 0.2677 & .9999 \\
\hline Convex & 0.9977 & 0.3433 & .9996 \\
\hline No fins & 0.9130 & 0.2155 & .9999 \\
\hline \multicolumn{4}{|c|}{ Experiment 2} \\
\hline Concave & 0.7960 & 0.3643 & .9956 \\
\hline Convex & 0.9340 & 0.4000 & .9996 \\
\hline \multicolumn{4}{|c|}{ Experiment $3 \mathrm{~A}$} \\
\hline Concave & 0.8507 & 0.2373 & .9999 \\
\hline Convex & 0.9850 & 0.3103 & .9996 \\
\hline \multicolumn{4}{|c|}{ Experiment 3B } \\
\hline Concave & 0.9670 & 0.3043 & .9999 \\
\hline Convex & 1.0240 & 0.6435 & .9999 \\
\hline
\end{tabular}

Note-For each of the four experiments, regression lines were fitted to stimulus span means for each version (averaging across other factors). The equation for each regression line contained an exponent that specified the slope $(a)$ and an exponent that specified the intercept $(b)$ in degrees of visual angle. $R^{2}$ indicates the degree of fit of the regression line to the data.

not a line segment (shaft) is present. On occasion, however, we may specify that a shaft was used, and may talk about "shaft length." Finally, most of the studies have used a fin set at each end of the span. We will describe the two members as a fin pair and designate the pair as being concave or convex according to their mutual relation to the span being judged.

\section{Depth Theories}

Gregory (1963) has argued that the distortions of length that characterize the Müller-Lyer illusion are due to misapplication of size constancy to the spans being judged. Specifically, he argues that the oblique orientations of the fin components serve as linear perspective cues, thus implying depth in an image that is, in fact, flat. When the fin pair is concave, the span that lies between the vertices is perceived as lying closer to the observer, and, when the fin pair is convex, the span is seen as being farther away. Under the influence of these depth cues, the observer then applies size-constancy rules to the spans, contracting the one that is enclosed by the concave fin pair and expanding the one that is enclosed by the convex pair. Thiéry (1896) first suggested this approach, and similar concepts are advanced by Fisher (1967).

We do not think it productive to review the various articles that argue against the depth/size-constancy hypothesis, and we would prefer to keep the focus on how it might apply to the present experimental results. The concept seems to require the existence of an "object," which is seen as lying closer to the observer or farther away than is true. This makes some sense if a line segment is present but becomes less plausible when the configuration shows a single fin set and the subject is asked to judge the open space between a dot at the vertex of that fin set and a reference dot.
However, giving the benefit of the doubt, let us assume that the dot is localized in the plane of the page and the fin set provides linear perspective cues that serve to shift the perceived depth of this component. With a concave configuration, the vertex dot might be seen as lying closer to the observer, and, with a convex configuration, the vertex dot might appear to be farther away. If so, then one would predict a gradient in the space to be judged, tilted most at the vertex and then progressing back (or forward) to meet the plane of the page at the location of the reference dot. If one then applies size-constancy principles, one would need to adjust for proximity of perceived elements, causing contraction of the concave span and expansion of the convex.

It is not clear, however, how the model would explain the results in the first two experiments, when the response span was indicated on the opposite side of the vertex. For a concave configuration, is the vertex dot seen as lying closer to the observer when the stimulus span is judged and then farther away when the judgment is rendered to the other side? This would require the depth perception to reverse as attention was shifted from one side of the fin set to the other. Alternatively, if one assumes that the fin set has no effect when the subject makes the response, one still has a problem with our finding that the error levels depend on the span being judged. If the fin set is tilting the vertex dot out of the plane of the page, then the two versions should produce symmetrical distortions as a function of distance. One would not expect the present results, wherein the size of illusion error for the two versions was opposite as a function of distance.

One might note also that depth theories depend heavily on concepts from projective geometry that appear to be at odds with our linear model for metric distortion. This is discussed below.

\section{Averaging Theories}

Several theories share the concept that the fin pair interferes with the perceptual system for metric encoding (i.e., measuring span). These include the concept of assimilation-first advanced by Brunot (1893) and extended by Pressey $(1967,1970)$, among others-which holds that a judgment is based on the average of distances enclosed by the fin pair. Delboeuf (1893) thought that the fin pair would attract the regard of the observer from the proper span to be judged, and Coren (1986) has adopted this approach. We include also the theory of Erlebacher and Sekuler (1969), who propose that the observer may confuse (and, thus, average) the distance between the tips of the fin pairs with the span that should be judged.

Most of the averaging theories presume a fin pair, with a fin at each end of the span providing a boundary of adjacent space, or targets of eye movement, or intertip distances. A single fin set does not contain some of the elements required by these theories, so one might be tempted to dismiss them as being misguided or irrelevant to the present analysis. We note, however, that Pressey (1970) 

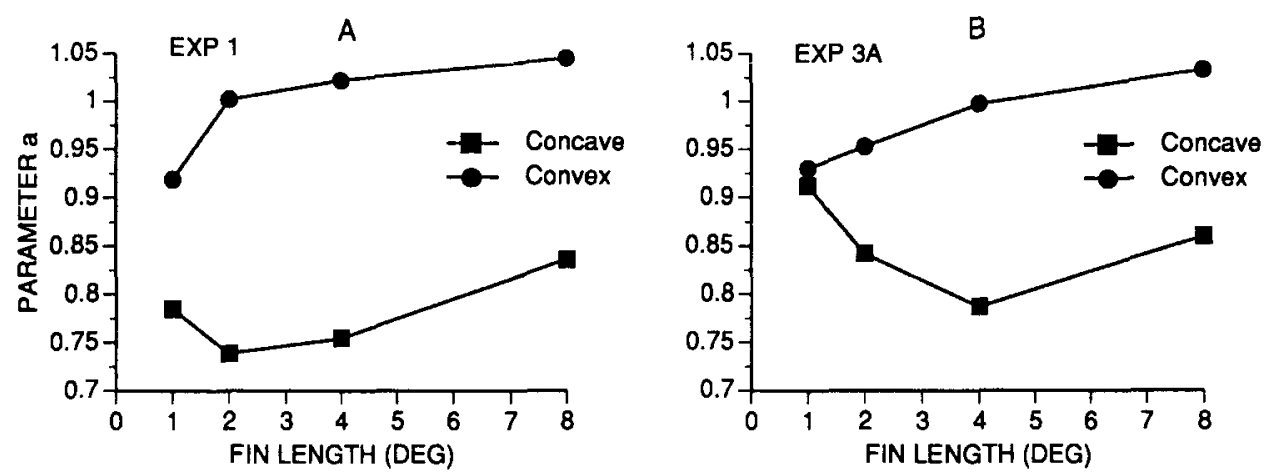

C
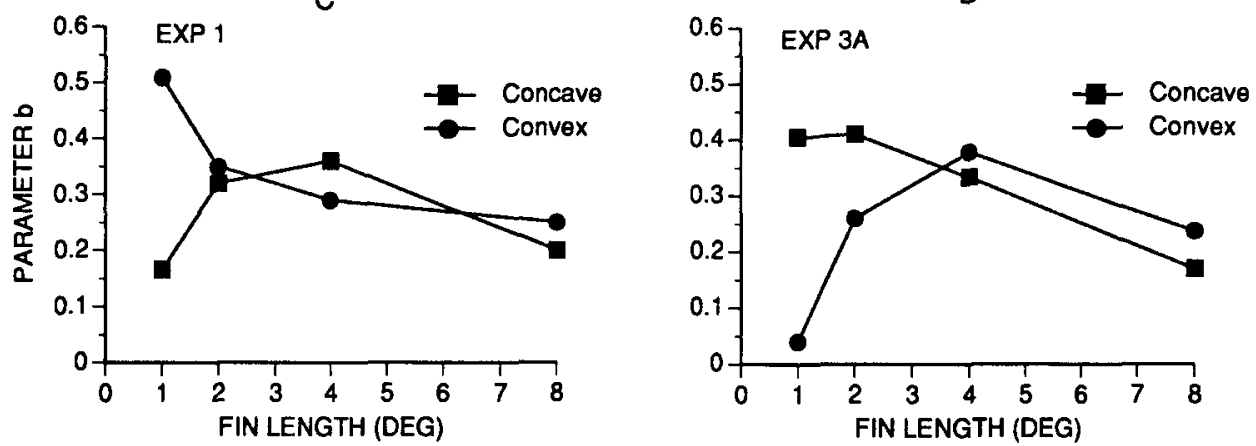

Figure 12. Additional insight is provided by fitting the linear equation to the means of Experiments 1 and $3 A$ at each level of fin length and then plotting the change in the value of the equation coefficients. In Panels $A$ and $B$, the a parameter (which specifies slope) has been plotted against fin length using a linear scale for both the ordinate and the abscissa. In Panels $C$ and $D$, the $b$ parameter (which specifies intercept) is plotted. The results support the conclusion that changes in fin length produce asymmetrical effects for concave and convex configurations.

provides an adaptation of the assimilation theory that posits that misperception of span may be based on measures taken between a fin set and a single reference dot. One might allow for similar adaptation of other averaging theories, so that a single fin set would serve to bias eye scans or other metric operations in relation to the reference dot. With this adaptation, the net effect should be in the same direction as when a fin pair is used-namely, contraction for concave and expansion for convex configurations. The only difference would be in the absolute magnitude of the predicted effect, with smaller errors for a single fin set than with a configuration that is bounded by a fin pair.

In general, therefore, the averaging theories argue that the stimulus span is not correctly judged because of other distances which influence the encoding process. These other distances are most discrepant when the stimulus span is short; therefore, one would expect illusion magnitude to be large at close distances and to decrease as stimulus span increases. However, in the present experiments, the reverse was found for concave configurations (i.e., strength of illusion increased with increasing stimulus span; see Figures $3 \mathrm{~B}, 5 \mathrm{C}, 8$ ). This was not a meager effect; rather, illusion strength was small or negative for the 1 deg span and grew to upwards of $20 \%$ when the span being judged reached $8 \mathrm{deg}$.
Nor is it feasible to salvage the averaging theories by rejecting the percent-error results in favor of our linear model. As indicated above, the model specifies two sources of distortion, one that is proportional to span and another that adds a constant. The values derived for the former were consistently less than unity for concave and convex configurations alike, which means that this factor describes a net contraction of the perceived span. For convex angles, the degree of contraction is relatively small and approaches unity as the convex angle becomes large (as seen in Figure 13). Nonetheless, it seems clear that effects that have been classically described as "expansion" of the perceived space are due to the addition of a constant error, as specified by the b parameter. "Expansion" by the addition of a constant is not consistent with the various averaging theories, which would require the perceptual summary to increase as the angle of the fins became progressively larger.

\section{Displaced Vertex Theory}

Chiang (1968) proposed that the perceptual system miscalculates the location of the vertex of the fin segments, displacing it toward the concave side. Thus, when a span to the concave side is evaluated, it is foreshortened by the amount of the displacement. Similarly, judgment of spans that lie on the convex side must add this distance. 

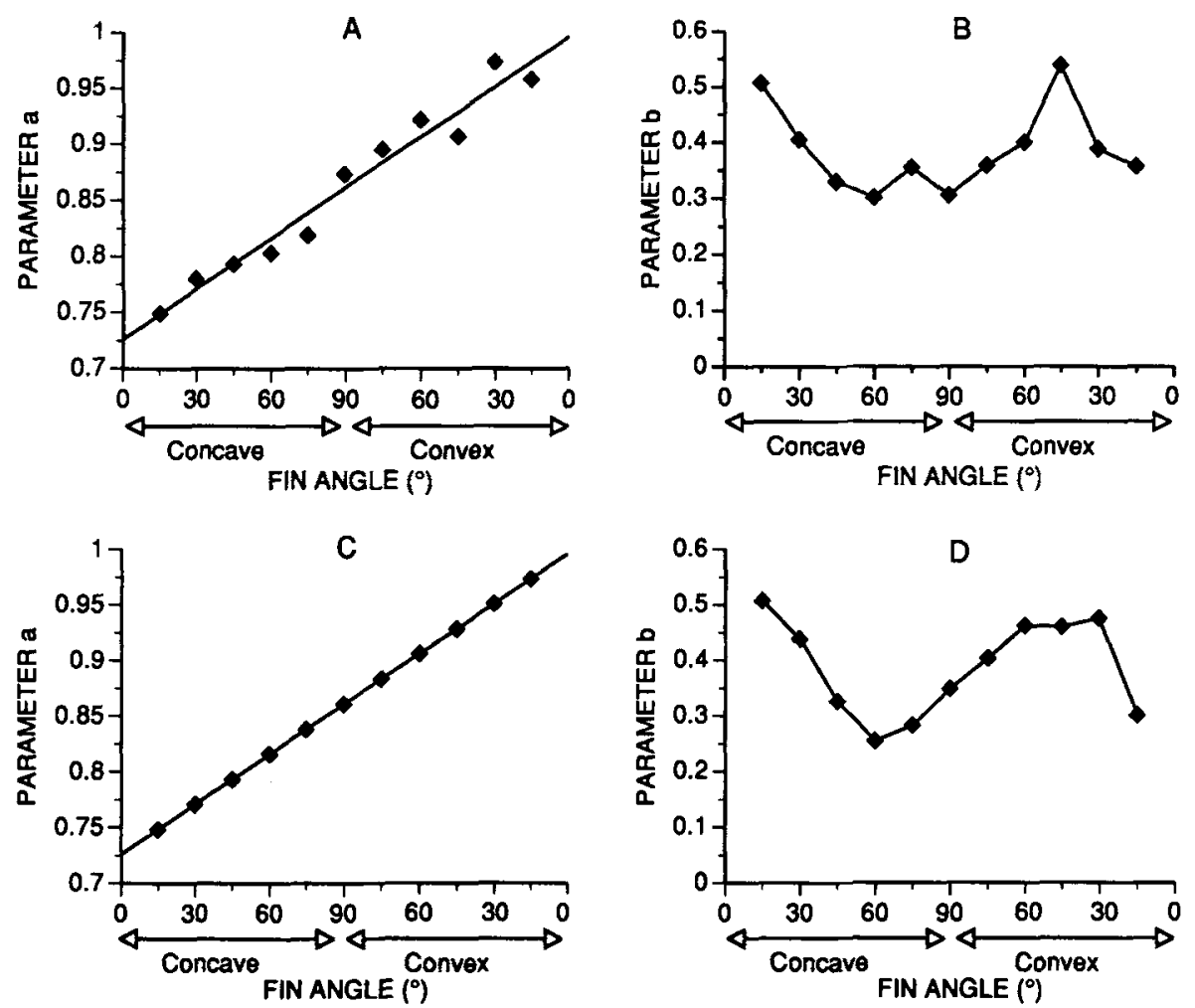

Figure 13. To further examine the influence of fin angle, the equation coefficients have been extracted from the data of Experiment 2 and are plotted in the upper panels. (A) The value of the $a$ parameter appears to be a linear function of angle across the entire range, and a regression line has been fitted. (B) The changes in the $b$ parameter do not suggest a simple functional relation. ( $C$ and $D$ ) If the values of the $a$ parameter are forced to the regression line, the resulting $b$ values show a sinusoidal oscillation as a function of angle.

In other words, his hypothesis is that $S^{\prime}=S \pm c$, wherein $c$ is the distance between the true location and the perceived location of the vertex. The displacement is sensitive to the relative angle between the fins in the set, being greater at close angles and going to zero as the fins become collinear (i.e., at $90^{\circ}$ ).

It might appear that Chiang (1968) has provided at least one element of our linear equation, taking the value of $c$ to be comparable to our $b$ parameter. However, it should be noted that all of the $b$ values extracted from our data were positive whether the configuration was concave or convex. Chiang would require $c$ to be negative with concave configurations, reflecting displacement of the vertex toward the inside of the convexity and thus producing a shortening of perceived span. With consistently positive $b$ values, it appears that this factor always expands the perceived space; thus, any contraction must come from the contribution of the a parameter. This tradeoff of effects allows for the possibility that concave configurations will produce "negative illusions" under some circumstances, and, indeed, expansion at short stimulus spans was a consistent feature of our results.

\section{Prior Studies of Stimulus Span}

The most important experimental result was the finding that the two Müller-Lyer versions produce differential effects as a function of the span being judged. It appears that this factor has been badly neglected in prior studies. Even among the parametric experiments that control viewing distance, many have presented only a single shaft length (e.g., Day \& Dickinson, 1976; Nakagawa, 1958; Pressey, 1974; Pressey \& Martin, 1990; Sekuler \& Erlebacher, 1971). Others have sampled within a very restricted range. For example, Erlebacher and Sekuler (1969) compared configurations having 200- or $212-\mathrm{mm}$ shafts ( 5.7 and $6.0 \mathrm{deg}$ ) and found the influence of manipulated angle to be comparable. Restle and Decker (1977) varied shaft length in several experiments but did not summarize their data in a way that would clearly reflect a differential effect for distance. In their Experiment 1 , they used shaft lengths of 19.6 and $23.1 \mathrm{~mm} \mathrm{(2.2}$ and $2.6 \mathrm{deg}$ ) and found a complex pattern of illusion strength as a function of fin length and angle. On the basis of the appearance of the plots, they suggested that the differences for shaft length were minimal. In Exper- 
iment 5, they used 3.5-, 7-, and 14-mm shaft lengths $(0.4$, 0.8 , and $1.6 \mathrm{deg}$ ), again varying fin length and angle. They did not find reliable treatment differences for the three shaft lengths and describe the effects as being "comparable." Two additional experiments were conducted that varied shaft length, but they used very unusual configurations, which makes it difficult to interpret their effects.

Given the considerable theoretical emphasis on the ratio of fin-to-shaft length, we find it difficult to understand why shaft length per se has not been manipulated over a larger range of values and with more attention to extracting the effects of this variable. Heymans (1896) may have set the stage for this trend, by claiming that the ratio of fin length to shaft length determined the strength of illusion effect. In fact, he had not manipulated shaft length as an independent variable; his conclusions were based on manipulation of fin length with shaft length being held constant or with both being varied simultanously to provide a constant ratio. It appears that other investigators accepted his claims as sufficient basis to ignore shaft length as a variable.

Morgan, Hole, and Glennerster (1990) used a suitable range of shaft lengths but presented their data in a way that is difficult to interpret. Specifically, they studied the influence of convex fin pairs using 2-, 4-, and 8-deg shaft lengths (designated as $D$ ), which were cross-hatched at different distances from the fin sets to designate the span to be judged. They found that illusion magnitude (expressed as $D^{\prime}$ divided by $D$ ) was a monotonic declining function of percent shaft length. No statistical tests were provided, but their figure shows a comparable linear decrease of effect for each of the three shaft lengths. Even if the pattern of the plots is accepted as being self-evident, it is not clear how one would interpret the plot of percent illusion against percent shaft length, the latter being the distance from the marker to the vertex of the fin set divided by shaft length. We read this as a plot of the metric distortion of the central shaft (being judged) against the metric distortion of the remainder of the shaft and have no idea what this ratio would signify. They suggest that it shows a gradient of effect from each fin set, a conclusion that is clearly at odds with the linear model.

Warren and Bashford (1977) used a variety of configurations having a fin set at each end of the shaft to be judged, as well as some that had a single fin set at one end of the shaft. In addition to asking subjects to mark a reference line to indicate the apparent length of the shaft, they also had them mark the stimulus shaft into four perceptually equal sections. The latter procedure is of special interest, since an uneven division of the shaft might reflect a differential action of the fin set(s) as a function of distance. Warren and Bashford provide tabled values for the degree of distortion for each quarter-section of the line, but it is difficult to reach any conclusions for a number of reasons: (1) The marking of the shaft into four quarters proceeded as a bisection, followed by bisection of each remaining half. The use of a two-stage procedure complicates the issue of how to conceive of the value for a given quarter-span. (2) They do not use any statistical test to establish whether one should reject the null hypothesis. (3) The pattern of effects is not very consistent across configurations that would be expected to show similar action by the fin set. Perhaps the authors were mindful of some of these problems, since they made no comment on differences among the four quarters for most of the configurations that they tested. They did claim a differential (outside quarters showing a stronger effect than did central quarters) when the configuration contained concave fins at each end of the shaft, but not when the fin sets were convex. It is possible that their second bisection (producing outside vs. inside quarters) is similar to our "internal" procedure in Experiment 3B. The results from our experiment suggests fin bias from convex and concave configurations; if anything, the convex effects are stronger. Given the problems listed above, we doubt their conclusion that there are substantial differences of illusion effect.

We know of only one study that presages our results for stimulus span and that is not confounded by simultaneous change of fin length or other procedural problem. Goryo et al. (1984) examined (among other variables) shaft lengths subtending 4.5, 5.0, and $5.5 \mathrm{deg}$ of visual angle. Across the three values, they found that illusion error was an increasing function of shaft length for concave configurations and a decreasing function of length for convex. Thus, they show the basic " $X$ " pattern that is so conspicuous across all of our plots of version $X$ stimulus span. Although they used a very restricted range of lengths, they appear to have sampled near the crossing point for the concave and convex slopes and, therefore, were able to observe the differential.

\section{Influence of Fin Set on Response Span}

At the risk of overstating the obvious, we think it is significant that configurations that contained only a single concave or convex fin set produced classic MüllerLyer effects, which were basically opposite for the two versions. This was not a foregone conclusion at the outset of the study, given that most theories of illusion mechanism seemed to require the presence of a fin pair. A major source of uncertainty (in Experiments 1 and 2) came from asking that the response span be placed next to the fin set. Although these task demands are similar to what is required by the composite version, the latter has a fin pair at each end which might be essential for producing an effect.

Rather than belabor the vagaries of what might have been found, let us have the benefit of hindsight. The clearest picture is provided by the results of linear modeling, which suggest a similar influence of concave and convex versions whether or not the response span is adjacent to the fin set. Experiments 1 and 3 A provide the most meaningful data, in that the task variables were comparable except for the placement of the response. The best-fit lines for Experiment 1 (Figure 13A) show more separation than 
do those of Experiment $3 \mathrm{~A}$ (Figure 13C), meaning that the distortion is greater when the subject must respond in the zone that is adjacent to the fin set. In other words, requiring the response to be in the space adjacent to the fin set increases the measure of distortion, which supports the speculation that the fin set acts to bias the metric of the space on both sides.

If one accepts this argument, then we must clarify and, perhaps, correct any cognitive errors that derive from the terminology which was adopted at the outset. Specifically, for efficiency (and before we had any idea of what kind of effects would be seen), we described the bias as a distortion of perceived stimulus span, as though the fin set had no influence on the response zone. Most likely, this is not the case, and a more realistic description of the process might be as follows (using a concave stimulus span as the prototype). The subject examines the stimulus span; the perception is biased, with the resulting encoded value for the span being smaller than is true. The subject must now use that encoded value to render a response span. If a remote location is provided (as in Experiment $3 \mathrm{~A}$ ), we might presume that it is metrically neutral, and the encoded value will not be subject to additional bias. This is not the case, however, if the subject is asked to use the zone that is adjacent to the fin set (i.e., on the convex side of the fin set). Here, the space being used for the response has been subjected to a reverse influence on its metric properties. On inserting the encoded span into the (perceptually) expanded space, the value is subject to additional shrinkage; therefore, the final (objectively measured) response span is found to be even shorter, as in Experiment 1.

We can think of two reasons why this analysis might not match one's intuitions. First, it presumes that the bias should be expressed as a change in the raw metric, as indicated by the linear modeling. If one conceives of the effects as reflected by percent errors, then the contraction of distances on the concave side of the fin set increases with distance, and the expansion on the convex side decreases with distance. One then has a potential paradox, in that the lack of influence on one side would be offset by strong influence on the other, yielding a net effect that is the same irrespective of stimulus span. The linear model shows that the distortions to the concave and convex side are the same with respect to distance, being shifted in proportion to the span that is judged and by the addition of a constant.

The second potential source of confusion relates to the insertion of an encoded (perceived) span into a space that is itself subject to distortion. Let us assume that a stimulus span lying on the concave side is perceived (encoded) as being shorter than is true, and the subject then is asked to respond to the convex side of the fin set. One might think that the fin set would act directly upon the encoded value, essentially "reexpanding" it to the original size. Instead, it appears that the fin set acts to expand the perception of the zone into which the encoded value must be inserted, so this value is further contracted in relation to the perceived dimensions of this zone.
Given the argument above, one might expect that use of the fin-adjacent site would produce illusion effects that were twice as large as when the response site was more remote. However, there is no reason to expect that the sources of bias are additive. If the upper limit of illusion effect is considered to be $100 \%$, then the bias that is generated on one side of the fin set might be $75 \%, 90 \%$, or $95 \%$; in other words, the bias from encoding the stimulus span already may be near the maximum level. If this is the case, then using the zone that is on the opposite side of the fin set will add to the effect, but the result will not be radically different from what is seen when the response zone lies at a distance.

\section{Interpreting a Linear Model}

In thinking about the metrics of visual space, we are shaped by concepts from projective geometry and physics. From projective geometry, we have the principle that dimensions should be interpreted as a function of distance from the observer, the transformation following a geometric progression known as the tangent rule. This means that the retinal dimensions of a stimulus decrease geometrically with displacement of the stimulus from the observer, with $\tan \alpha=$ span/distance providing a good approximation of the change. We know that the retinal image of what we see is (functionally) flat and that our perception of depth is derived mainly from alignments and sizes within the stimulus itself. Therefore, it is reasonable to wonder whether the metric distortion in visual images might be due to misinterpretation of the cues for depth.

From physics, we have the concept of a "force field," which involves a change in strength of effect as a function of distance. The interactions at any given location must spread to have an influence at other sites, and effects decrease as an inverse function of distance. We have learned that such forces can modify the metric of physical space itself, which thus leads naturally to Riemannian-like theories in which the position or orientation of line segments can induce changes in the metric of visual space.

It is clear, therefore, that physical force fields are characterized by a gradient (usually a geometric progression), and depth theories rely on a geometric change in values for interpreting the span of a stimulus. This is not the relationship that is called for by the present linear model of metric distortion. Here, the multiplying parameter $a$ and the additive parameter $b$ are applied irrespective of the distance that is judged, and there is no gradient for the effect. What may be confusing here is the fact that the $a$ parameter introduces a distortion that is proportional to length, but, with further reflection, it should be clear that the derivative of the equation (which specifies the gradient) is a constant. Thus, the "strength" of the effect does not become weaker as the length is increased, and the changes in the $a$ and $b$ parameters do not follow a tangent rule, as would be required to specify depth.

The notion that specific equation coefficients (determined by the characteristics of a given fin set) will adjust any span that is displayed should not disturb a reader who is disposed to a top-down approach to perception. If 
the focus is on a particular judgment, one can accept any lawful rule for how it might be changed. However, for those of us who view the process as requiring preconscious interactions among a myriad of lines and points, the challenge is to provide a picture of how the extended metric is simultaneously affected. A "linear distortion" cannot be applied simultaneously across the entire extent of the visual field, because to do so would provide unbiased judgments when the response zone lies in the open field (i.e., away from the fin set). Perhaps the reference dot provides a boundary for the action of the fin set, such that "bias of stimulus span" describes not just a change of encoded value but a uniform rescaling of the metrics within this portion of visual space.

\section{REFERENCES}

ADAM, J., \& BATEMAN, L. (1983). A correlational analysis of symmetry between the arrowhead and featherhead Müller-Lyer illusions. Perception, 12, 119-129.

AUERBACH, F. (1894). Erk! ̈̈rung der Brentano's optischen Täuschung [Explanation of Bentano's optical illusion]. Zeitschrift für Psychologie, 7, 152-160.

BEAGLEY, W. K. (1985). Interaction of Müller-Lyer with filled-unfilled space illusion: An explanation of Müller-Lyer asymmetry. Perception \& Psychophysics, 37, 45-49.

BRENTANO, F. (1892). Über ein optisches Paradoxon [Concerning an optical paradox]. Zeitschrift für Psychologie, 3, 349-358.

Bross, M., Blair, R., \& Longtin, P. (1978). Assimilation theory, attentive fields, and the Müller-Lyer illusion. Perception, 7, 297-304.

BRUNOT, C. (1893). Les illusions d'optique [Optical illusions]. Revue Scientifique, 52, 210-212.

Butchard, N., \& Pressey, A. W. (1971). The effects of closing lines on the two forms of the Müller-Lyer illusion. Psychonomic Science, 23, 257-258.

Chiang, C. (1968). A new theory to explain geometrical illusions produced by crossing lines. Perception \& Psychophysics, 3, 174-176.

Christie, P. S. (1975). Asymmetry in the Müller-Lyer illusion: Artifact or genuine effect? Perception, 4, 453-457.

COOPER, M. R., \& RuNYON, R. P. (1970). Error increase and decrease in minimal form of the Mueller-Lyer illusion. Perceptual \& Motor Skills, 31, 535-538.

COREN, S. (1970). Lateral inhibition and geometrical illusions. Quarterly Journal of Experimental Psychology, 22, 274-278.

COREN, S. (1986). An efferent component in the visual perception of direction and extent. Psychological Review, 93, 391-410.

COREN, S., \& Porac, C. (1983). The creation and reversal of the MüllerLyer illusion through attentional manipulation. Perception, 12, 49-54.

DAY, R. H., \& DiCKINSON, R. G. (1976). Apparent length of the arms of acute and obtuse angles, and the components of the Müller-Lyer illusion. Australian Journal of Psychology, 28, 137-148.

DeLBoEUf, J. (1892). Sur une novelle illusion d'optique [On a new optical illusion]. Bulletin de l'Academie Royal de Belgique, 24, 545-558.

DEL BOEUF, J. (1893). Une nouvelle illusion d'optique [A new optical i1lusion]. Revue Scientifique, 51, 237-241.

DEWAR, R. E. (1967). Stimulus determinants of the magnitude of the Müller-Lyer illusion. Perceptual \& Motor Skills, 24, 708-710.

Erlebacher, A., \& Sektler, R. (1969). Explanation of the MüllerLyer illusion: Confusion theory examined. Journal of Experimental Psychology, 80, 462-467.
Erlebacher, A., \& Sekuler, R. (1974). Perceived length depends on exposure duration: Straight lines and Müller-Lyer stimuli. Journal of Experimental Psychology, 103, 724-728.

FISHER, G. H. (1967). A common principle relating to the Müller-Lyer and Ponzo illusions. American Journal of Psychology, 80, 626-631.

Goryo, K., Robinson, J. O., \& Wilson, J. A. (1984). Selective looking and the Müller-Lyer illusion: The effect of changes in the focus of attention on the Müller-Lyer illusion. Perception, 13, 647-654.

GREGORY, R. L. (1963). Distortion of visual space as inappropriate constancy scaling. Nature, 199, 678-680.

Greist-Bousquet, S., \& SCHiffman, H. R. (1981a). The many illusions of the Müller-Lyer: Comparisons of the wings-in and wingsout illusions and manipulations of standard and dot forms. Perception, 10, 147-154.

Greist-Bousquet, S., \& SchiffMan, H. R. (1981b). The role of structural components in the Mueller-Lyer illusion. Perception \& Psychophysics, 30, 505-511.

Heymans, G. (1896). Quantitative Untersuchungen über das "optische Paradoxon" [Quantitative studies of the "optical paradox"]. Zeitschrift für Psychologie, 9, 221-225.

JoRdAN, K., \& UHLARIK, J. (1986). Length contrast in the Müller-Lyer figure: Functional equivalence of temporal and spatial separation. Perception \& Psychophysics, 39, 267-274.

LEWIS, E. O. (1909). Confluxion and contrast effects in the Müller-Lyer illusion. British Journal of Psychology, 3, 21-41.

Morgan, M. J., Hole, G. J., \& Glennerster, A. (1990). Biases and sensitivities in geometrical illusions. Vision Research, 30, 1793-1810.

Müller-Lyer, F. C. (1889). Optische Urteilstäuschungen [Optical i]lusions]. Archiv für Anatomie und Physiologie: Physiologische Abteilung, 2 (Suppl.), 263-270.

Nakagawa, D. (1958). Müller-Lyer illusion and retinal induction. Psychologia, 1, 167-174.

Pressey, A. W. (1967). A theory of the Müller-Lyer illusion. Perceptual \& Motor Skills, 25, 569-572.

PREsSEY, A. W. (1970). The assimilation theory applied to a modification of the Müller-Lyer illusion. Perception \& Psychophysics, 8, 411-412.

Pressey, A. W. (1974). Effect of size of angle on the ambiguous MüllerLyer illusion. Acta Psychologica, 38, 401-404.

Pressey, A. W., \& MarTin, N. S. (1990). The effects of varying fins in Müller-Lyer and Holding illusions. Psychological Research, 52, 46-53.

Restle, F., \& DeCKer, J. (1977). Size of the Mueller-Lyer illusion as a function of its dimensions: Theory and data. Perception \& Psychophysics, 21, 489-503.

SEKUlER, R., \& ERLEbacher, A. (1971). The two illusions of MüllerLyer: Confusion theory reexamined. American Journal of Psychology, 84, 477-486.

THIÉRY, A. (1896). Über geometrisch-optische Täuschungen [Concerning geometric optical illusions]. Philosophische Studien, 12, 67-125.

WARREN, R. M., \& BASHFORD, J. A. (1977). Müller-Lyer illusions: Their origin in processes facilitating object recognition. Perception, 6, 615 626.

Weintraub, D. J., \& Brown, S. (1986). Perceptual plane geometry: Collinearity judgments probe the perceived orientation of an angle's sides. Journal of Experimental Psychology: Human Perception \& Performance, 12, 434-444

WORRALL, N., \& FirTh, D. (1974). The components of the standard and reversed Müller-Lyer illusions. Quarterly Journal of Experimental Psychology, 26, 342-354.

(Manuscript received August 3, 1995; revision accepted for publication April 4, 1996.) 